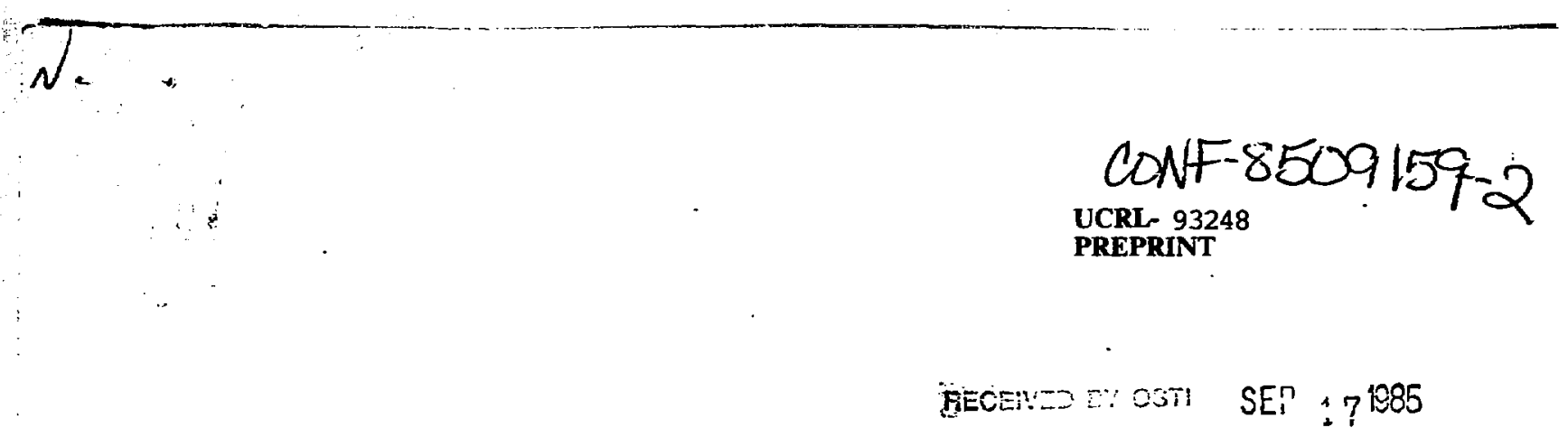

\author{
PRECOMPOUND NUCLEON ANGULAR DISTR IBUTIONS \\ IN THE CONTINUUM
}

M. Blann, W. Scobel, and E. Plechaty

Lawrence Livermore National Laboratory

University of California

Livermore, CA 94550

This paper was prepared for submittal to

the 17th International Summer School

on Nuclear Physics

Mikolajki, Poland

September 2-14, 1985

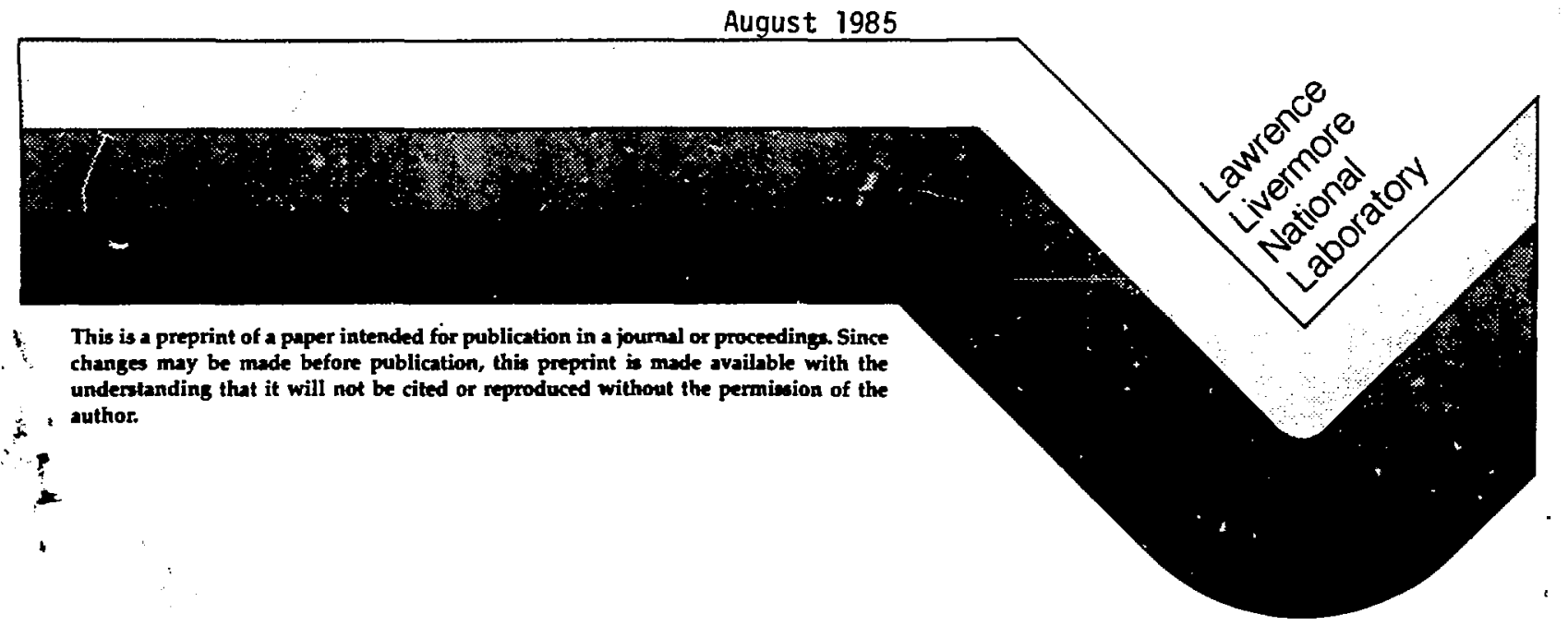

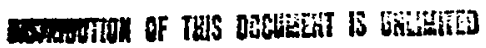




\title{
DISCLAIMIXR
}

This report was prepared as an acoount of wock sponsoced by an seacy of the Uaitod States Government. Neither the United Stites Grernmeal nor any atency thereof, nor any of their

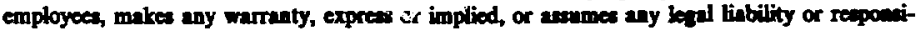
bility for the sccuracy, completesces, or usefulness of any informalioe, apparatus, product, or process diselowed, of represents that its use would not infringe privetely ownod rights. Reference herein to any specific commercial product, proceses, or service by trade name, tradeanark. manufacturer, or otherwive does not nocescarily constitute or imply its endornement, recommendation, or faroring by the Unitod Stales Government or any aseacy thereof. The views and opinions of authors expreaned herein do not necestarily state or refloct thowe of the Unitod Stales Government or any apeacy thereof.

\section{PRECOMPOUND NUCLEON ANGULAR DISTRIBUTIONS IN THE CONTINUUM}

M. Blamn, W. Scobel, + and E. Plechaty

Lawrence Livermore National Laboratory

University of California

UCRL--93248

Livermore, CA 94550

DE85 017832

\begin{abstract}
Angular distributions for mucleon induced reactions (incident energies 14 to $90 \mathrm{MeV}$ ) leading to precompound muclecn emission in the continum (emitted particle energies 9-70 MeV are calculated based on mucleon-nucleon scattering kinematics for an incident nucleon on a Fermi gas. Analytic expressions due to Kiluchi and Kawni are used for the single scattering kernel. The geometry dependent hybrid model is used to generate the differential cross sections for first, second, etc. order scattering, these weightings being used to fold the single scattering kernel. Results are found to reproduce all experimental angular distribution quite well at angles in the $20^{\circ}$ to $90^{\circ}$ range. Ad-hoc modifications to approximate quantal effects and Coulomb deflections are explored, but the results do not seem to offer a consistent means of reproducing back angle yields, and give generally poorer results at very forward angles.
\end{abstract}

\section{NTRSUUCTION}

The goal of the present work is to consider the interpretation of angular distributions of mucleons emitted in the contimum for reactions induced by $14^{\circ}$ to $90 \mathrm{MeV}$ mucleons. This will be done in terms of mucleon-mucleon scattering kinematics between an

+ Permanent address: 1. Institut fir Experimentalphysik, Univ. Hamburg, Hamburg. Federal Republic of Germany.

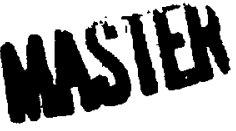


M. BLANN

incident mucleon and target mucleons having a Fermi momentum distribution. Some of the geometric (local density) information as well as single versus multiple scattering fractions and emission cross sections will be provided by using the geometry dependent hybrid model ${ }^{2}$ (GDH) in conjunction with nucleon-nucleon scattering kinematics for a projectile scattering with nucleons having a Fermi gas energy distribution. ${ }^{2}$ We will attempt to place the resuits of this work in perspective with other closely related approaches, such as the intranuclear iscade model, ${ }^{3-3}$ and more recent and rigorous approaches involving quantum mechanical procedures. ${ }^{5,7}$ We will also mention exciton model approaches whicin precede this work. 12 The main new contribution of this work will be use of the GDH to give multiple scattering folding fractions. However we will also be critical in looking at both existing quantum mechanical and semiclassical treatraents on this subject, and believe that some of the conclusions of this work may lead to perspectives on the subject which differ from those of some of the earlier works.

To our knowledge Goldberger (1948) presented the first analytic expressions for the angular distribution of a nucleon after scattering with nucleons having a Fermi gas momentum distribution, including restrictions due to the Pauli exclusion principle. ${ }^{20}$ This approach was repeated by Hayakawa, Kikuchi and Kawai (1955) who considered examples of (p,p') and (p,n) reactions at $18 \mathrm{MeV}$ incident proton energy. ${ }^{24}$ These authors included estimates of refraction for incident and emitted particles, with trajectories based on impact parameter and the mean free path for mucleon-mucieon (N-N) scattering. Only a single N-N scattering process was considered.

Intramuclear cascade models predicted angular distributions based on $N-N$ scattering kinematics, and included multiple 


\section{PRECOMPOUND NUCLEON ANGULAR DISTRIBUTIONS}

scattering processes (which was not done in Refs. 13,14). Some of the newer INC codes did include a semiclassical treatment of reflection and refraction.

Exciton type models were first used by Mantzouranis et al." as a means of estimating single versus multiple scattering contributions to use in folding a kernel based on N-N scattering kinematics. A $90^{\circ}$ scattering approximation was made in deriving an analytic expression for the kernel, resulting in a loss of part of the kinematic information. This point is lllustrated in a more quantitative manner in Sec. 3 of this work.

Sun Ziyang et al." utilized the analytic expressions of Hayakawa et al. ${ }^{12}$ as reported by Kikuchi and Kawai ${ }^{2}$ to provide a N-N scattering kernel for folding using the exciton model, thereby maintaining the kinematics due to the Fermi momentum distribution. However these authors used a kernel which was averaged over all final nucleon energies, thereby losing the information of the differences expected in angular distributions between nucleons losing small amounts of energy versus those losing large amounts of energy. Akkermans and Gruppelaar ${ }^{10}$ first followed the metl.od of Mantzouranis et a1., and later ${ }^{22}$ of Sun Ziyang et al. "They explored the consequences of adding refraction first in the entrance channel, and then also in the exit chamel. $^{22}$ More recently they have used the Hayakawa analytic expressions to give the energ; dependent angular distribution of particles following the first scatiering process, folding with an energy averaged result for muitiple scattering processes. ${ }^{2}$ (These authors carefully considered errors due to use of an energy averaged kernel for scattering beyond the first collision, and demontrated that the approximations made were very reasonable.)

Several quantum mechanical formulations of the problem have been presented. Tamura et ㅆ.1." do a wave mechanical 
M. BLANN

treatment of single and double $N-N$ scattering processes with interference among intermediate two quasiparticle states. A comprehensive presentation of this approach was recently published in this journal." A somewhat less rigorous approach has been presented by Feshbach et a‥ ${ }^{7}$ In this approach a single scattering kemel is calculated using DWBA; a statistical argument is then invoked to fold this result incoherently, somewhat analogous to the folding of the semiclassical $N-N$ scattering kernel by Mantzouranis et al. " Unfortumately there appear to be some errors and/or ambiguities in the application of this model, and a correct calculation with this theory remains to be performed. 13,26

In Section 2 of this work we present a very brief formulation of the geometry dependent hybrid model, followed by a description of the calculation of the angular distribution for a single nucleon-nucleon scattering event in nuclear matter according to Ref. 14, and of the folding of this kernel for multiple scattering. In Section III we will cornpare results using the N-N scattering kernel, folded for multiple scartering processes, with experimental data for reactions induced by incident nucleons of 14.7 to $90 \mathrm{MeV}$. In Section IV we will consider ad-hoc methods of treating quantum mechanical effects in the entrance and exit channels via semiclassical approximations, and discuss possible shortcomings of the approximations in the calculational model of folding of the N-N scattering kernel. Our conclusions are given in Section V; they are intended to be critical in order to focus attention on those aspects of the subject which we believe should be considered open and awaiting improvement, and areas where we think caution is required lest we are headod up wrong paths. 


\section{PRECOMPOUND NUCLEON ANGULAR DISTRIBUTIONS}

\section{FORMULATIONS TO CALCULATE $N-N$ SCATIERING}

DISTRIBUTIONS AND TO WEIGHT THEIR FOLDING

\subsection{Hybrid and Geometry Dependent Hybrid Models}

In this work we explore the applicability of $\mathrm{N}-\mathrm{N}$ scattering kinematics in reproducing the observed angular distributions of (nucleon in, nucleon out) reactions. The single scattering result for $\mathrm{N}-\mathrm{N}$ scattering in a Fermi gas was derived analytically by Goldberger $^{23}$ and later by Hayakawa et al. ${ }^{24}$ We will try to extend this result to include multiple $\mathrm{N}-\mathrm{N}$ scattering contributions. The vehicle to weight single versus multiple scattering results will be the geometry dependent hybrid model (GDH), ${ }^{2}$ with some comparisons also using the hybrid model. ${ }^{2} 7$

Use of exciton type models (including hybrid or GDH) to predict angular distributions is in a sense a contradiction, for these models owe their simplicity to the abandonment of angular information in their derivation. However these models follow energy partition according to the number of $\mathrm{N}-\mathrm{N}$ scattering collisions, and therefore may be used to give the weighting, for a given exit channel energy, between one, two, three, etc. collision contributions. In addition to the $\mathrm{N}-\mathrm{N}$ scattering events which are assumed to be represented as incoherent processes in semiclassical exciton models, we also will consider (as did Hayakawa et al. ${ }^{12}$ and later Costa et a1. ${ }^{12}$ ) shape-elastic (coherent) processes such as refraction of the incident projectile and emitted ejectile.

Nucleon-mucleon scattering kinematics will be dependent on the locai density of the collision site; incident particle refraction will depend upen entrance chamel impact parameter, and local density of the trajectory. Exit channel refraction will depend upon collision position, angle and local density of trajectory, information which is totally missing in exciton model formulations 


\section{BLANN}

(including hybrid and GDH models). We will return to this point in Section 4.

The hybrid and GDH models have recently been reviewed in some detail. ${ }^{27}$ We very briefly present formulations here for completeriess of discussion, and refer to Ref. 17 for a more complete description.

The hybrid model has been formulated as

$$
\begin{gathered}
P_{v}(\epsilon) d \epsilon=\sum_{n=n_{0}}^{\bar{n}}\left[n X_{v} N_{n}(\varepsilon, U) / N_{n}(E)\right] \varepsilon \\
\left.\cdot\left\{\lambda_{c}(\epsilon) / \lambda_{c}(\epsilon)+\lambda_{+}(\epsilon)\right]\right\} D_{n} d \epsilon
\end{gathered}
$$

where symbols are defined in Table $I$. The differential cross seation is given by

$$
\frac{\dot{d} \sigma}{d \epsilon}=\sigma_{\mathbf{R}} P_{v}(\epsilon)
$$

In order to reproduce local density effects to first order, the hybrid model was reformulated as a sum over each incident entrance channel partial wave $2,{ }^{2}$

$$
\frac{d \sigma}{d \epsilon}=\pi \lambda^{2} \sum_{\ell=0}^{\infty}(2 \ell+1) T_{\ell} P_{v}^{\ell}(\varepsilon)
$$

where Fermi energy and particle mean free paths are evaluated in a local density approximation over an assumed (unrefracted) entrance channel trajectory. Fermi density distributions are used for the averaging process, with a sharp radius based on the Myers

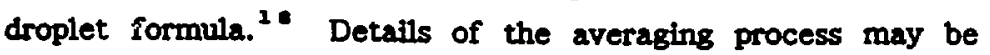
found elsewhere. ${ }^{2}$

It may be seen that the GDH formulation preserves more of the physical information necessary for treating the $N-N$ 


\section{PRECOMPOUND NUCLEON ANGULAR DISTRIBUTIONS}

\section{TABLE I. Definition of symbols}

\begin{tabular}{|c|c|}
\hline Symbol & Definition \\
\hline$P_{v}(\varepsilon)$ de & $\begin{array}{l}\text { number of particles of the type } v \text { (neutrons or } \\
\text { protons) emitted into the unbound continumum with } \\
\text { channel energy between } \epsilon \text { and } \epsilon+d \epsilon \text { (MeV). }\end{array}$ \\
\hline$\overline{\mathbf{n}}$ & $\begin{array}{l}\text { equilibrium (most probable) particle plus hole } \\
\text { (exciton) number. }\end{array}$ \\
\hline$n_{0}$ & initial exciton number. \\
\hline$n^{x_{v}}$ & $\begin{array}{l}\text { number of particles of type (proton or neutron) } v \text { in } \\
\text { an n exciton hierarchy. }\end{array}$ \\
\hline $\mathbf{E}$ & composite system excitation \\
\hline $\mathbf{U}$ & residual nucleus excitation. \\
\hline $\mathrm{N}_{\mathrm{n}}(\epsilon, \mathrm{U})$ & $\begin{array}{l}\text { number of ways that } n \text { excitons may be combined } \\
\text { such that one, if emitted, would have channel energy } \\
\epsilon \text { and the remaining } n-1 \text { excitons would share } \\
\text { excitation } U=E-B_{U}-\epsilon \text {, where } B_{v} \text { is the particle } \\
\text { binding energy. }\end{array}$ \\
\hline $\mathbf{N}_{\mathbf{n}}(\mathrm{E})$ & $\begin{array}{l}\text { number of combinations with which n excitons may } \\
\text { share excitation energy } \mathrm{E} \text {. }\end{array}$ \\
\hline$\lambda_{c}(\epsilon)$ & $\begin{array}{l}\text { emission rate of a particle into the continunum with } \\
\text { channel energ } \varepsilon \text {. }\end{array}$ \\
\hline$\lambda_{+}(\varepsilon)$ & $\begin{array}{l}\text { intranuclear transition rate of a particle which would } \\
\text { have channel energy } \epsilon \text { if it were emitted into the } \\
\text { continwum. }\end{array}$ \\
\hline$D_{n}$ & $\begin{array}{l}\text { fraction of the initial population which has survived } \\
\text { to an n-exciton hierarchy. }\end{array}$ \\
\hline$\sigma_{R}$ & reaction cross section. \\
\hline \& & orbital angular momentum in units $\hbar$. \\
\hline$x$ & reduced de Broglie wavelength. \\
\hline$\epsilon$ & channel energy \\
\hline $\mathbf{B}$ & single particle state density \\
\hline
\end{tabular}




\section{BLANN}

scattering and incident refraction questions than a hybrid/exciton model approach in which all density dependencies are averaged out. For this reason we will primarily use the GDH model for calculation of angular distributions in this work. Even in this formulation the necessary information for exit channel refraction is totally missing, and we will ultimately use a quite arbitrary means of handling this problem.

\subsection{Nucleon-Nucleon Scattering Kinematics}

2.2.1. Basic scattering kernel. In this subsection we present the result of Hayakawa, Kikuchi and Kawai ${ }^{2,14}$ for the angular distribution of nucleons following a single nucleon-nucleon scattering event in nuclear matter as a function of incident and final nucleon energies and Fermi energy. The expressions exclude scattering events for which either scattered nucleon has an energy less than the Fermi energy. The result is derived on the assumption that the total nucleon-nucleon scattering cross section is independent of energy. ${ }^{2.14}$ Calculations with a more reasonable 1/E dependence of $\mathrm{N}-\mathrm{N}$ scattering cross sections yield much more complicated results ${ }^{14}$; Kikuchi and Kawai suggest that the spectral shapes are not significantly different for results using the more realistic 1/E energy dependence. ${ }^{2}$

The results of Hayakawa et al.. as taken from the later work of Kikuchi and Kawai, are represented by

$$
\frac{d^{2} \sigma}{d E_{1} d \Omega}=\frac{3 \sigma_{f}\left(E_{1}\right)}{8 \pi E_{F}}\left(\frac{E_{1}}{E_{F}}\right)^{1 / 2} \cdot \frac{1}{\sqrt{Q_{1}}}\left(E_{1}-E_{f}\right)
$$

or

$$
\frac{d^{2} \sigma}{d E_{I} d \Omega}=\frac{3 \sigma_{f}\left(E_{1}\right)}{8 \pi E_{F}}\left(\frac{E_{1}}{E_{F}}\right)^{1 / 2}
$$




\section{PRECOMPOUND NUCLEON ANGULAR DISTRIBUTIONS}

$$
\text { - } \frac{1}{Q \sqrt{Q E_{1}}}\left[E_{1} E_{Y} \sin ^{2} \theta-Q\left(E_{Y}-E_{F}\right)\right]
$$

where $\sigma_{f}\left(E_{1}\right)$ is the total cross section of free $N-N$ collisions at the incident laboratory enerby of the particle $E_{1}$ in nuclear matter, $E_{F}$ is the Fermi energy, $E_{f}$ is the final nucleon energy after scattering, and where

$$
Q=E_{1}+E_{f}-2 \sqrt{ } E_{1} E_{f} \cos \theta
$$

[Our Eq. (4a) differs from the result of Ref. 13 by a factor of 2 , which we believe was a type-setting error in Ref. 13.] The energy $E_{1}$ is measured from the bottom of the Fermi sea. Whether to use (4a) or (4b) is determined by the following: if

$$
\begin{array}{lll}
E_{f}<E_{1}-E_{F}, & \alpha_{2}<\cos \theta<\beta_{2}, & \text { use (4b) } \\
E_{f}>E_{1}-E_{1} & \beta_{1}<\cos \theta<\beta_{2}, & \text { use (4b) } \\
& \alpha_{1}<\cos \theta<\beta_{1} \quad \text { use (4a) } \\
& \alpha_{2}<\cos \theta<\alpha_{1} \quad \text { use (4a) }
\end{array}
$$

where

$$
\begin{aligned}
& a_{1}=\left\{E_{1}-E_{F} \mp\left[E_{F}\left(E_{F}+E_{f}-E_{1}\right)\right]^{1 / 2}\right\} \vee E_{1} E_{f} \\
& \beta_{1} \\
& a_{2}=\left\{E_{f}-E_{F} \mp\left[E_{F}\left(E_{F}+E_{1}-E_{f}\right)\right]^{1 / 2}\right\} \vee E_{1} E_{f} . \\
& \beta_{2}
\end{aligned}
$$

The angular distributions for each final nucleon energy following a scattering process were calculated at 36 angles spaced $5^{\circ}$ apart, beginning at $2.5^{\circ}$ using Eqs. (4) and (5). For the hybrid 


\section{BLANN}

model calculation a maximum value of $E_{f}=40 \mathrm{MeV}$ was used; for the GDH calculacion the angular distribution was calculated for the maximum $\mathrm{E}_{\mathrm{f}}$ along each trajectory. For the incident channel of angular momentum $\ell$ with partial cross section $\sigma_{\ell}$, the kernel was weighted as $\sigma_{\ell} / \sigma_{R}$ (where $\sigma_{R}$ is the total reaction cross section) and the results were summed over all enirance channel partial waves to get a weighted first scattering kernel. This kernel was folded with a pure hybrid model kernel (i.e. $\mathrm{E}_{\mathrm{f}}=$ $40 \mathrm{MeV}$ ) for higher order scattering, since nucleons are no longer localized by impact parameter after an intranuclear scattering event. The folding of these histogram kernels is discussed in the next subsection.

It is instructive to look at results of Eqs. (4a-b) for the sirugle scattering of a $90 \mathrm{MeV}$ proton incident on a ${ }^{90} \mathrm{Zr}$ target nucleus, giving neutrons of final energy 5,50 and $80 \mathrm{MeV}$. These results are shown in Fig. 1. A Fermi energy of $40 \mathrm{MeV}$ was used in these calculations. Also shown in Fig. 1 is the single scattering result (kernel) due to Mantzouranis et al. in which the $90^{\circ}$ approximation (target nucleons stationary) was made. We see from Fig. 1 that the $90^{\circ}$ approximation limits scattering to $90^{\circ}$ or less, whereas inclusion of the target nucleon Fermi momentum permits larger angle scattering for lower enargy collision partners. Mantzouranis et al. restricted their derivation to the fast particles; however in popular usage of their model this restriction has sometimes been ignored. ${ }^{20,21}$ We see in Fig. 1 that the shapes of the angular distributions vary considerably with final energy; we do not therefore feel that the use of an $\mathrm{N}-\mathrm{N}$ kernel averaged over energy is desirable, consistent with the conclusion of Ref. 12. Note also (Fig. 1) the large difference in shape between the singie scattering kernel with the $90^{\circ}$ approximation versus the result of the more rigorous Fermi gas calculation according to Ref. 2 . 


\section{PRECOMPOUND NUCLEON ANGULAR DISTRIBUTIONS}

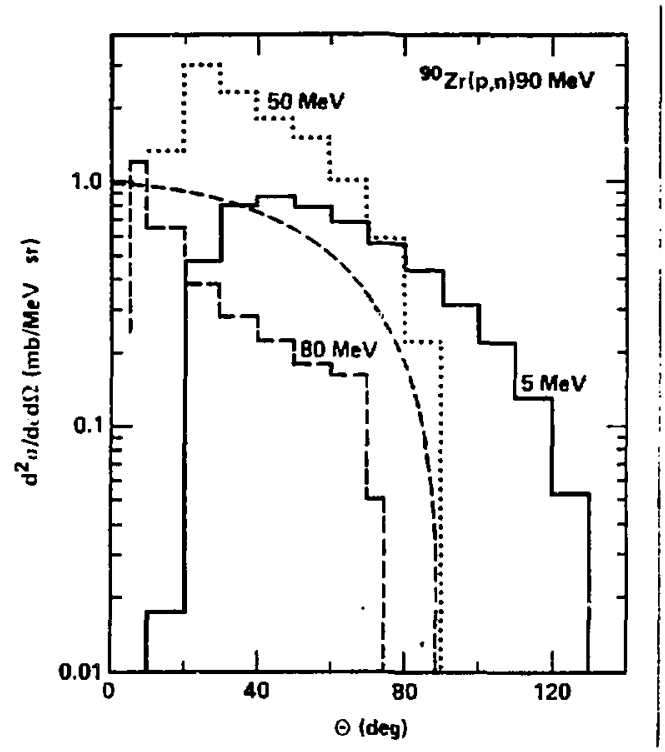

FIG. 1. Angular distribution kernels (single scattering event) for $90 \mathrm{MeV}$ protons incident on a Fermi gas with $\varepsilon_{\mathrm{f}}=40 \mathrm{MeV}$. The solid histogram is for $5 \mathrm{MeV}$ neutrons (laboratory reference) from a $10 \mathrm{Zr}$ target; dotted histograms for $50 \mathrm{MeV}$ neutrons, and dashed histogram for $80 \mathrm{MeV}$ neutrons. The smooth dashed curve is the result of the $90^{\circ}$ approximation used in Ref. 8.

Additional kinematic information is summarized in Fig. 2, where the minimum and maximum scattering angles are shown versus final scatiered nucleon energy for incident nucleons of 45 and $90 \mathrm{MeV}$ (a $40 \mathrm{MeV}$ Fermi energy was used in these calculations). It may be seen that e.g., for the $90 \mathrm{MeV}$ incident nucleon case, the maximum final angles vary between 128 and 68 degrees for collision partne rs receiving energies between 5 and $80 \mathrm{MeV}$ in the frame of the emitted nucleons. This figure re-emphasizes the point of the previous paragraph. This is of course the reason that Costa et $\underline{11}^{22}$ adopted an energy dependent kernel (as we will now do) rather than an energy averaged kernel as e.g., in Ref. 9. 


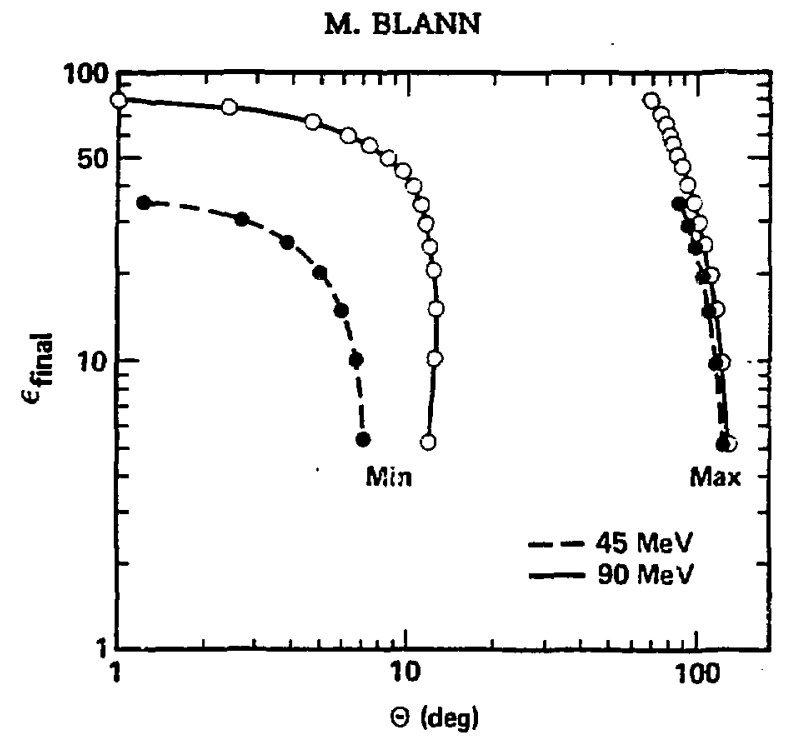

FIG. 2. Kinernatic limits for single nucleon-nucleon scattering events in a Permi gas. The dashed and solid curves represent the minimum and maximum angles versus final nucleon energy for $45 \mathrm{MeV}$ incident (dashed lines) and $90 \mathrm{MeV}$ incident (solid lines) nucleons and a Fermi energy of $40 \mathrm{MeV}$.

2.2.2. Folded kernel for multiple scattering. There are two points which require discussion in going from the single scattering kerriel described by Eqs. (4) and (5), to a result folded for second and higher order scattering. The first is the method of evaluating the kernel for a second scattering when the energy $E_{1}$ is not so simply defined as for the incident projectile, which is used for the primary scattering kernel. The second point is the method of folding on a jroper unit sphere, i.e., in three dimensional space.

We have made an approximation in evaluating the kernel for second and higher order scattering; we have assumed that the

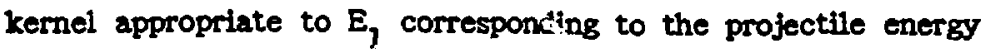
may be used, rather than a weighted suri: of kernels corresponding to a weighting of e.g. parent exciton populations which could 


\section{PRECOMPOUND NUCLEON ANGULAR DISTRIBUTIONS}

contribute to a given final exciton energy. Thert are reasons why we expect this to be a quite satisfactory approximation. The first is that for most cases we investigate, the first $(n=3)$ exciton term dominates the emission process, and for this term $E_{1}$ is unambiguously the projectile energy. Since higher order ( $n>3$ ) terms are in a sense a perturbation on the dominant first term, the error in using the primary kernel rather than a secondary-weighted kornel becomes a perturbation on a perturbation, i.e., a second order error.

Reference to Fig. 2 shows that whether $E_{1}$ is $45 \mathrm{MeV}$ or $90 \mathrm{MeV}$, the kinematically determined maximum scattering angles do not differ by any large amount for given final exciton energies. From ihis it may be inferred that use of the primary kernel should not, as argued above, cause a significant error.

Tests of a related nature for the point under discussion were made by Costa et al. ${ }^{12}$ who performed one set of calculations using the primary kernel as we have for all orders of folding. They then performed calculations using the primary kernel only for $n=3$, and a single kernel averaged over all emission energies for $n>3$. They found that the differences in final results between these extremes were small enough that they could comfortably use the energy averaged kernel for $n>3$. Quantitative estimates of differences between these two procedures may be found in Table Il of Ref. 12 .

We also note that use of a more rigorously consistent parent weighted scattering kernel would in all cases decrease the final maximum scattering angle for a given exit channel energy. Consequently the deficiencies we will observe in calculating back-angle yields cannot be attributed to this approximation which is in the opposite direction.

The second point involves the method of folding the kernel defined by Eqs. (4) and (5) for multiple scattering, or for attempts 


\section{BLANN}

to estimate quantum mechanical effects which may be another means of scattering the incoming particles. To do so we have used a subroutine which follows the direction cosines in spherical coordinates using a deterministic method as follows:

(1) The initial scattering angie $\theta_{1}$ about the beam direction is selected as $2.5^{\circ}$

(2) The initial azimuthal angle $\varphi_{1}$ is selected as $2.5^{\circ}$

(3) A scattering angle $\theta_{2}$ with respect to the direction $\theta_{1}$ is selected at $2.5^{\circ}$

(4) in azimuthal angle of $\varphi_{2}=2.5^{\circ}$ is selected.

The direction cosines are now computed with respect to the original beam direction. Next $\varphi_{2}$ is rotated in $5^{\circ}$ increments from $2.5^{\circ}$ to $357.5^{\circ}$, recomputing direction cosines for each case. This process is repeatis as $\theta_{2}$ is advanced from $2.5^{\circ}$ to $177.5^{\circ}$ in $5^{\circ}$ increments. The above processes are repeated as $\varphi_{1}$ is advanced from $2.5^{\circ}$ to $87.5^{\circ}$ (due to considerations of azimuthal symmetry), and as $\theta_{1}$ is advanced from $2.5^{\circ}$ to $177.5^{\circ}$. Wieighting factors of

$$
\frac{d^{2} \sigma_{1}}{d E_{1} d \theta} \sin \left(\theta_{1}\right)
$$

and

$$
\frac{d^{2} \sigma_{2}}{d \epsilon_{2} d \Omega} \sin \left(\theta_{2}\right)
$$

are multiplied to get a relative weighting of each element in this folding process. We are only interested in those elements which would be detected by e.g. a counter in a plane about the target. We therefore only process and store those events which fall into a range of $\pm 5^{\circ}$ about such a plane. As a check we expanded the \pm $5^{\circ}$ acceptance angle to $\pm 10^{\circ}$. No significant change in 


\section{PRECOMPOUND NUCLEON ANGULAR DISTRIBUTIONS}

angular distribution resulted. For folding of the scattering kernel to approximate quantal effects, $\theta_{1}$ (or $\theta_{2}$ ) is restricted to a range of angles which is based on quantal spreading limits we try to fold in, with a $\sin \theta_{1}\left(\right.$ or $\sin \theta_{2}$ ) weighting factor. The scattering kernel retains the

$$
\frac{d^{2} \sigma_{2}}{d \epsilon_{2} d \Omega} \sin \left(\theta_{2}\right)
$$

weighting, or the folded weighting factor for the appropriate angle (whether a primary kernel or folded result depends on the stage of the calculation, i.e., $n=3$ or $n>3$ ).

In the following section we will present results of the semiclassical N-IN scattering kernel, for multiple scattering using the GDH model of Eq. (3), with a few comparisons also with the hybrid model of Eq. (2). We mention that Mantzouranis performed a closed form hybrid model calculation ${ }^{20}$ using the kernel described in Ref. 8.

In Section 4 we will discuss some of the quantal effects which should cause spreading of the angular distributions beyond the range resulting from the nucleon-nucleon scattering kernel, and show results of some very ad-hoc semiclassical estimates of such effects. We will want to emphasize the very arbitrary nature of these add-ons to the $\mathbf{N}-\mathbf{N}$ scattering; nonetheless it is worth an attempt to improve deficiencies in the pure $\mathbf{N}-\mathbf{N}$ scattering approach for use in muclear technology, and perhaps as a probe of what success is possible in a semiclassical framework.

\section{RESULTS USING A FOLDED N-N SCATTERING KERNEL}

In Figs. 3-9 experimental $\left(n, n^{\prime}\right)$ and $(p, n)$ angular distributions are shown and compared with calculated folded $N-N$ scattering results using equations $(4 a-b)$ due to Kilauchi and Kawai with Eqs. (2) or (3). Data are from Refs. 23-27. 
M. BLANN

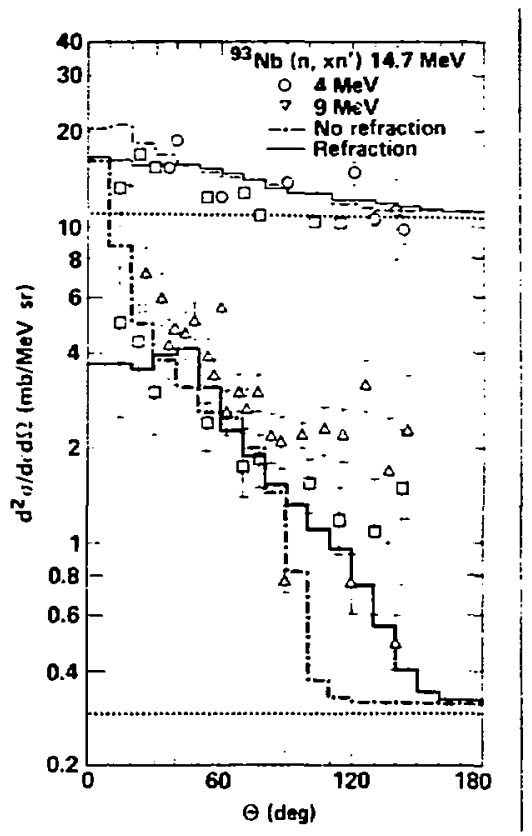

FIG. 3. Experimental and calcilated angular distributions for the - $\mathrm{Nb}(\mathrm{n}, \mathrm{xn})$ reaction with $14.7 \mathrm{MeV}$ incident neutrons. Results are shown for $4 \mathrm{MeV}$ and $9 \mathrm{MeV}$ emitted neutrons. Data (circles, squares and triangles) are from Ref. 23. The dotted lines are the calculated equilibrium components which have been added to the histogram results. The dot-dash histograms are the results of the semiclassical calculation using the folded $N-N$ scattering sertit described in the text. The solid histograms are the results of the same calculation but with an additional folding for 'refraction' in the entrance chamel as described in the text.

For the case of $14.7 \mathrm{MeV}$ incident neutrons (Fig. 3) the result of this calculation plus the assumed isotropic evaporation components (as given by the Alice/Livermore 82 code) are in quite satisfactory agreement with the experimental results. However a large part of the back angle yields is attributable to equilibrium evaporation, decreasing the sensitivity of the comparison to the shape of the the $\mathbf{N}-\mathbf{N}$ scattering kernel. 


\section{PRECOMPOUND NUCLEON ANGULAR DISTRIBUTIONS}

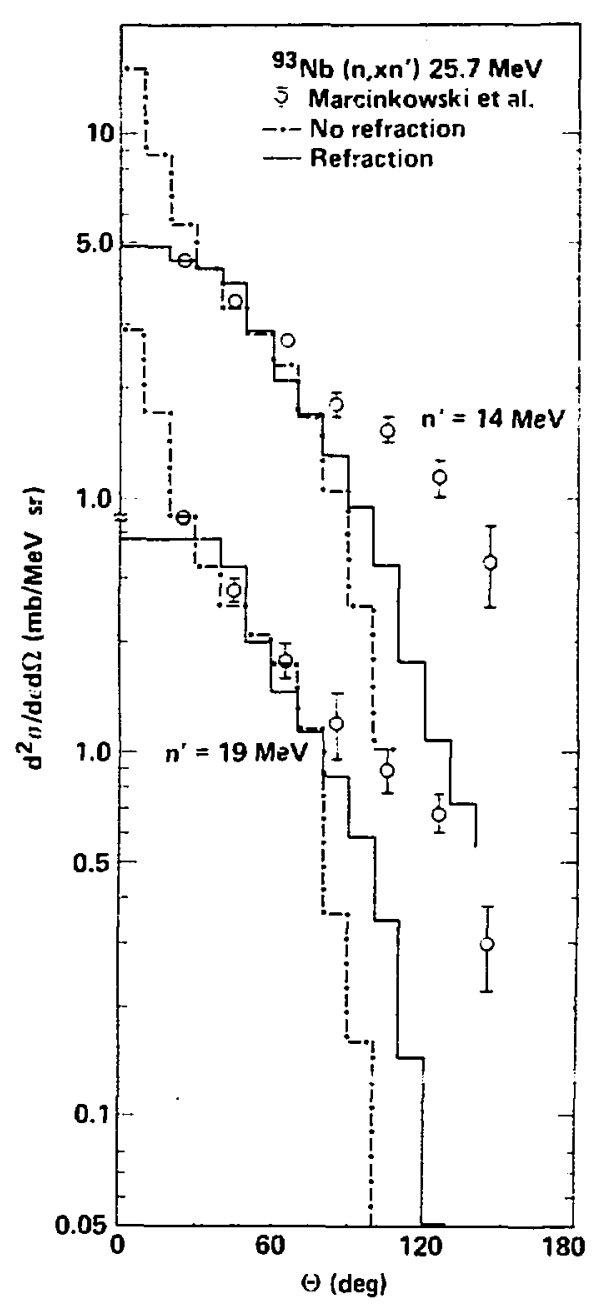

FIG. 4. Results as in Fig. 3 for an incident neutron energy of 25.7 MeV, for 14 and $19 \mathrm{MeV}$ exit channel energies. Data (circles) are from Ref. 24. The dot-dash histogram is the pure folded $\mathrm{N}-\mathrm{N}$ scattering result; the solid histogram includes the folding for entrance channel refraction described in the text. 


\section{BLANN}

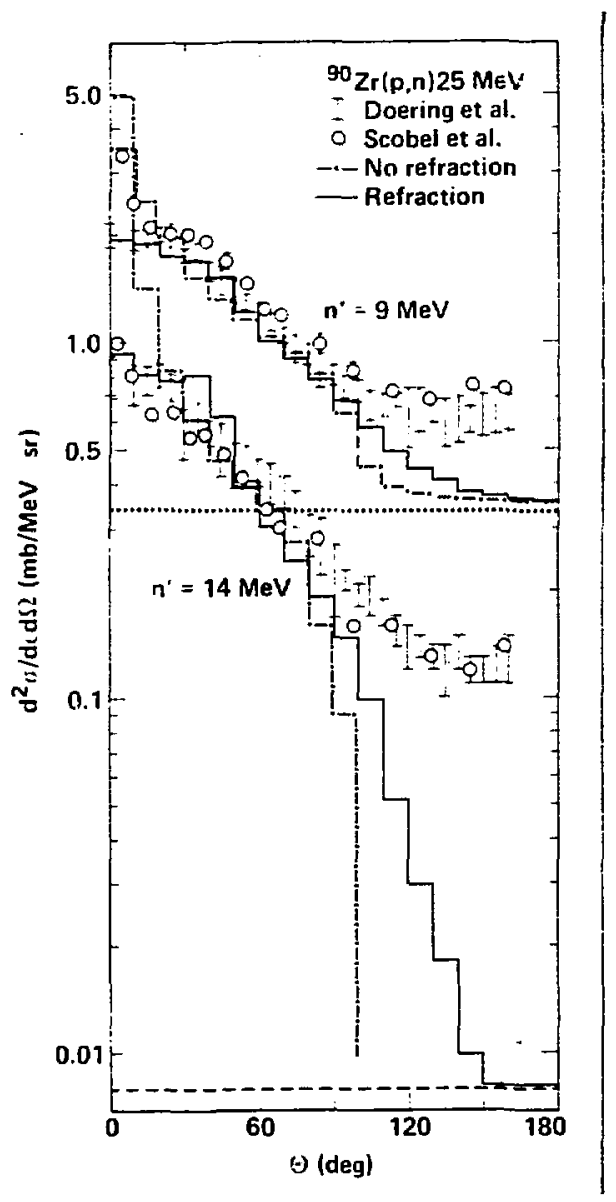

FIG. 5. Results as in Fig. 4 for the ${ }^{\circ} \mathrm{Zr}(p, n)$ reaction at $25 \mathrm{MeV}$ incident proton energy. Experimental results are from Ref. 22 and 25 (horizontal bars) and from Ref. 26 (circles). Calculated results are as in Fig. 4. The data of Refs. 20, 23 are more reliable than those of Ref. 24 at angles forward of $20^{\circ}$. The isotropic (dotted and dashed) components are the calculated evaporation contributions which have been acded to the precompound results shown. 


\section{PRECOMPOUND NUCLEON ANGULAR DISTRIBUTIONS}

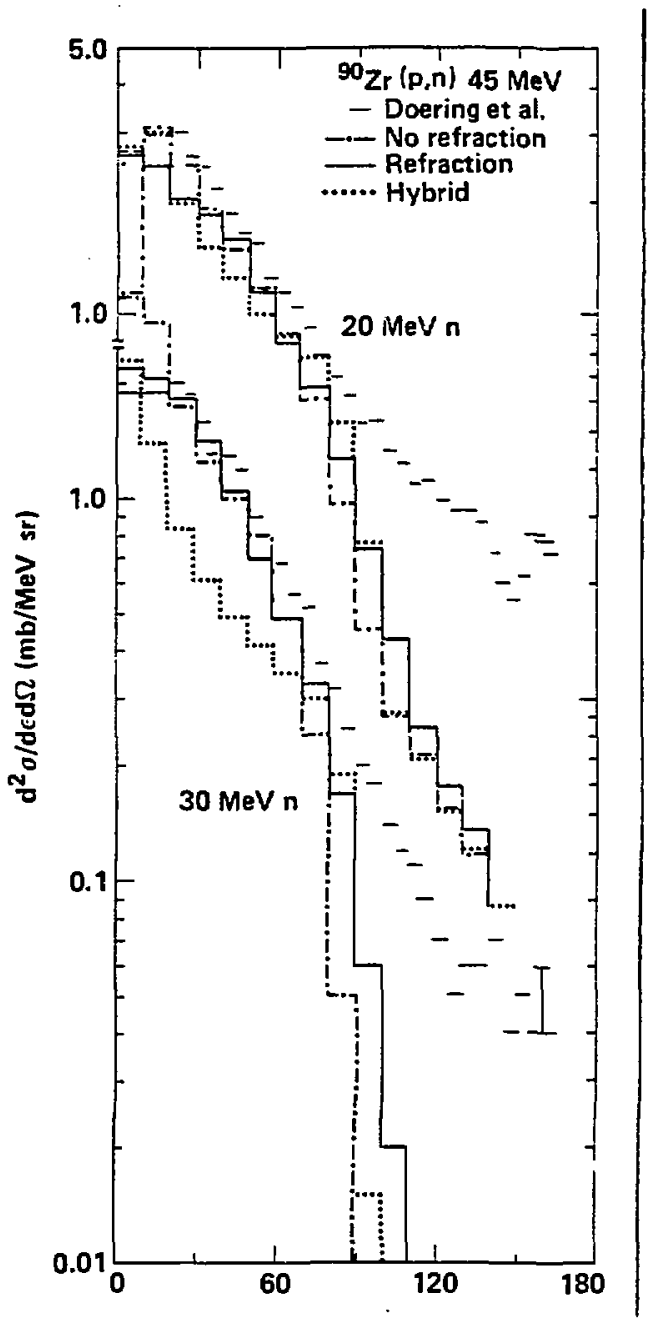

FIG. 6. As in Fig. 5 for an incident proton energy of $45 \mathrm{MeV}$. Data (horizontal bars) are from Refs. 22, 25. Calculated results are as in Fig. 4 for exit channel energies of 20 and $30 \mathrm{MeV}$. 


\section{BLANN}

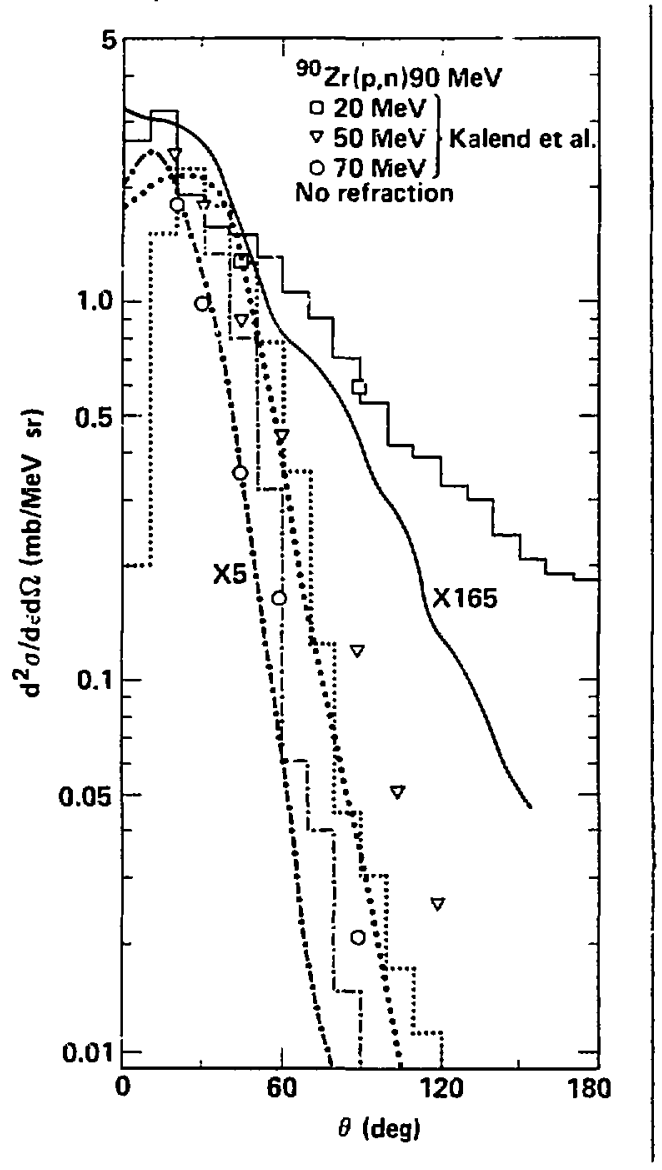

FIG. 7. As in Fig. 5 for an incident proton energy of $90 \mathrm{MeV}$. Data are from Ref. 27 for exit channel energies as shown. Solid curves have been drawn througin the data for 50 and $70 \mathrm{MeV}$ exit channel energies to guide the eye. Histograms are folded $N-N$ scattering results without refraction. The $20 \mathrm{MeV}$ exit channel result includes a significant equilibrium contribution. 
M. BLANN

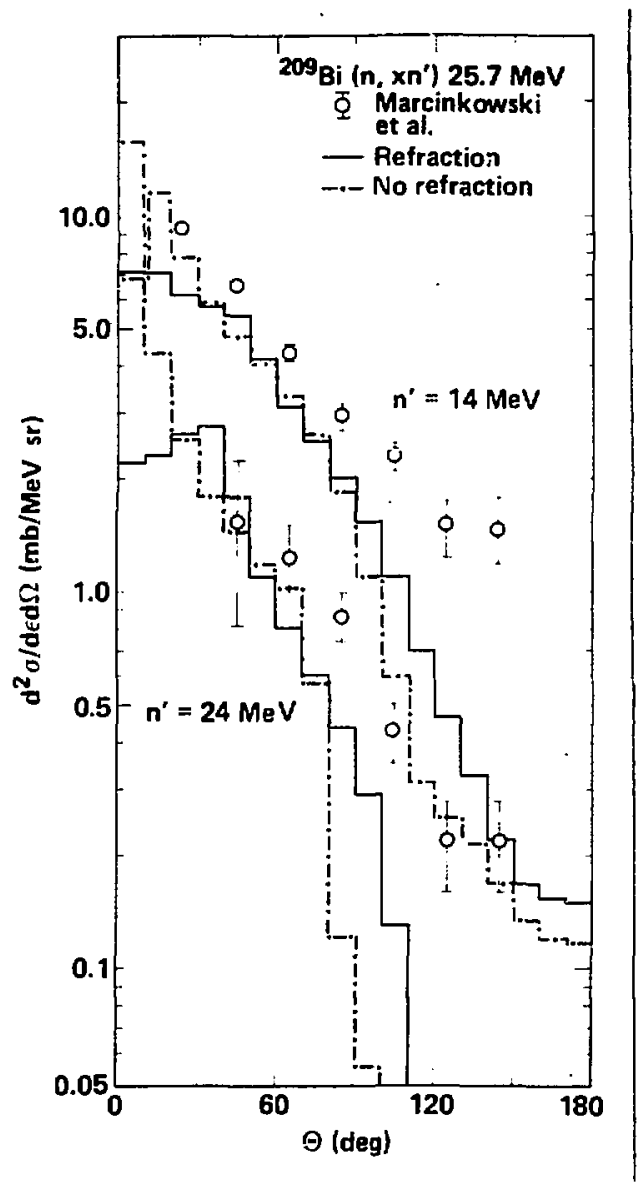

FIG. 8. As in Fig. 4 for the $209 \mathrm{Bi}(\mathrm{n}, \mathrm{xn})$ reaction with $25.7 \mathrm{MeV}$ incident neutrons. Data are from Ref. 24; calculated histograms are as in Fig. 4.

In Fig. 4 similar comparisons are shown for $25.7 \mathrm{MeV}$ incident neutron energy, and in Figs. $5-7$ for the ${ }^{\circ} \mathrm{Zr}(\mathrm{p}, \mathrm{n})$ reaction at incident energies of 25, 45 and $90 \mathrm{MeV}$. In Figs. 8 and 9 results are shown for ${ }^{200} \mathrm{Bi}(\mathrm{n}, \mathrm{xn})$ at an incident neutron energy of 25.7 MeV. Hybrid model results (Eq. 2) are shown in Figs. 6, 7 and 


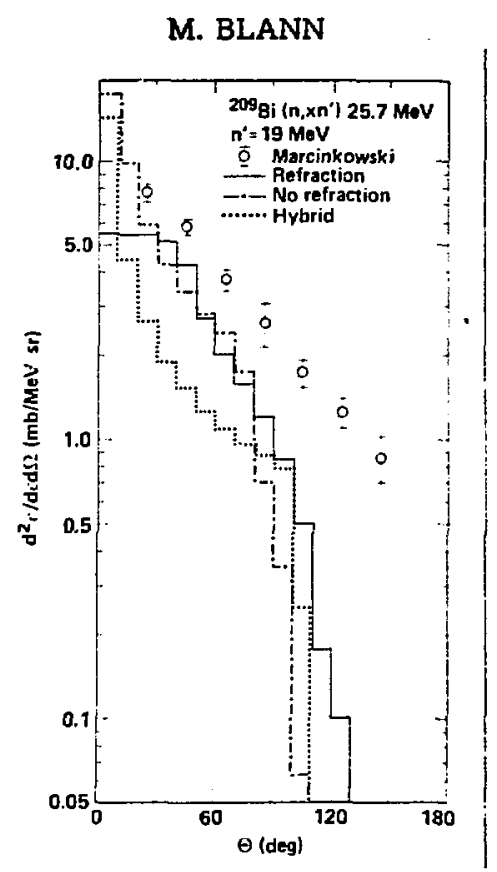

FIG. 9. As in Fig. 8 for $19 \mathrm{MeV}$ exit channel energy.

9. All of these results may be summarized as follows: the $N-N$ scattering kernel gives a very good representation of the slope of the angular distribution for all incident and exit channel energies at angles between $20^{\circ}$ and 80 to $100^{\circ}$ where single scattering dominates. Beyond this angle, as may be anticipated from Fig. 10 (see Section IV), there is insufficient yield predicted at far back angles. This is similar to the case of the intranuclear cascade calculation; results of the INC for the ${ }^{\circ} \mathrm{Zr}(p, n)$ reaction are shown in Fig. 11. Here the deviation between experimental and calculated results is large at $60^{\circ}$. An important point to bear in mind is that the shapes of the angular distributions calculated solely by $\mathrm{N}-\mathrm{N}$ scattering kinematics gives an excellent reproduction of the major part of the emission cross section for all exit 


\section{PRECOMPOUND NUCLEON ANGULAR DISTRIBUTIONS}

energies. While the discrepancies at back angles in Figs. 3-9 may appear large on a semi-log plot, the cross section involved is very small. The important point is that if one is seeking angular distribution information for technology, the result using the folded kernel of $\mathrm{N}-\mathrm{N}$ scattering is quite good. The comparisons in Figs. 3-9 are un-normalized, and obviously give good absollice agreement with data (in the range $20^{\circ}$ to $80-100^{\circ}$ ) when the GDH model gives a good resilt for the angle integrated cross section. We observe in Figs. 6, 7 and 9 that the shape of the angular distribution resulting from the GDH model is better than that resulting from the hybrid model.

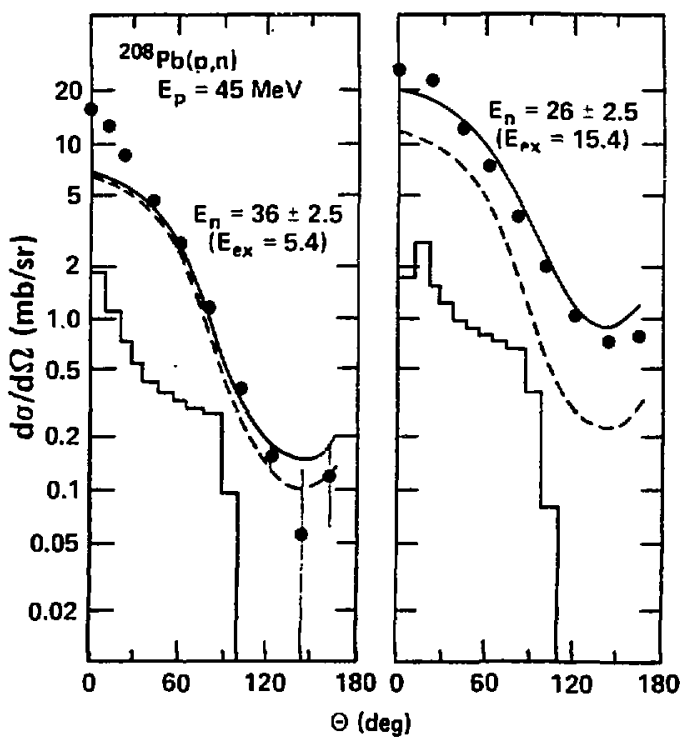

FIG. 10. Angular distributions for $20 \cdot \mathrm{Pb}(\mathrm{p}, \mathrm{n})$ at $45 \mathrm{MeV}$ incident energy. The data (circles) are from Ref. 22; the dotted curve:; the single scattering result of Ref. 6 , and the solid curve the result of single plus double scattering according to the theoretical result of Ref. 6 . The solid histogram is the semiclassical single scattering result due to Eqs. (4-5) based on the work of Refs. 2 . 13 , and 14. 
M. BLANN

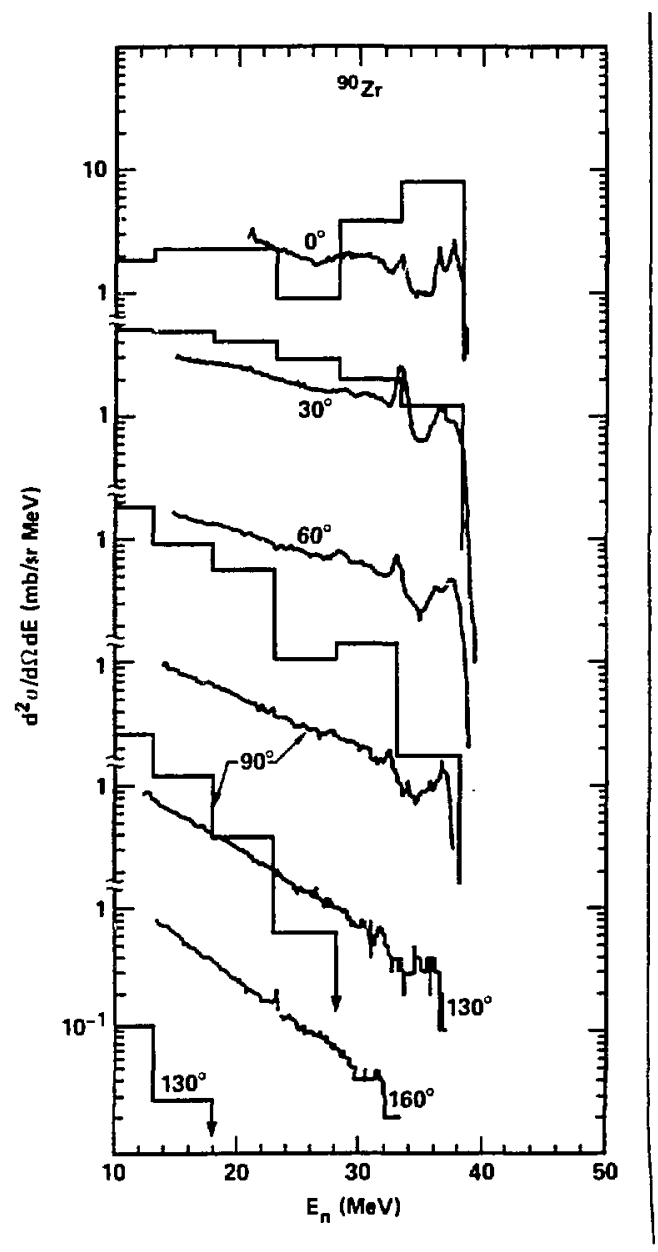

F1G. 11. Experimental and calculated $0^{\circ} \mathrm{Zr}(p, n)$ spectra versus angle of measurement for $45 \mathrm{MeV}$ incident protons. The calculated results are based on the intramuclear cascade model and are from Ref. 29. The histogram is the cascade model result; solid irregular lines represent experimental results. 


\section{PRECOMPOUNE NUCLEON ANGULAR DISTRIBIJTIONS}

We feel that the conclusions above give the main essence of physics of the $\mathrm{N}-\mathrm{N}$ scattering approach to the yuestion of continum angular distributions in the precompound region. The comparison with the results of Tamuri (Fig. 10), and the information of Fig. 12 (to be discussed in Section IV), suggests that if one wishes to reproduce the small cross section at back angles as well, a more rigorous quantum mechanical treatment is required. Alternatively one may explaits scattering into the backward hemisphere in a semiclassical model and in agreement with both energy and momentum conservation, if part of the linear momentum is transferred to a heavier system. In this case the energy transfer is negligibly small. On these grounds, there has been a tendency to attempt to modify the semiclassical treatment described thus far by ad-hoc addition of semiclassical effects intended to approximate quantal phenomena. Such procedures become very tenuous; they are valuable and legitimate to gain physical insights. However, our personal viewpoint is that attempts to interpret them beyond the 'insight' stage must be made with extreme caution. Having stated this caveat we will explore such 'add-ons' to the nucleon-nucleon scattering approach.

\section{OUANTAL EFFECTS AND SEMI-CLASSICAL APPROXIMA- TIONS}

In this section we will discuss constraints on nucleon angular distributions due to diffraction and refraction. Diffraction is a puraly quantum mechanical phenomenon which has no semiclassical analogue. It is related to nuclear size and nucleon wave number. Refraction has a semiclassical limit, but it is questionable tiat this limit is reached in nuclear reactions in the energy range of interest in this work. Refraction will depend on the change in potential between the medium and the vacuum, and on the geometry of the rucleon trajectory. However, exciton 
M. BLANN

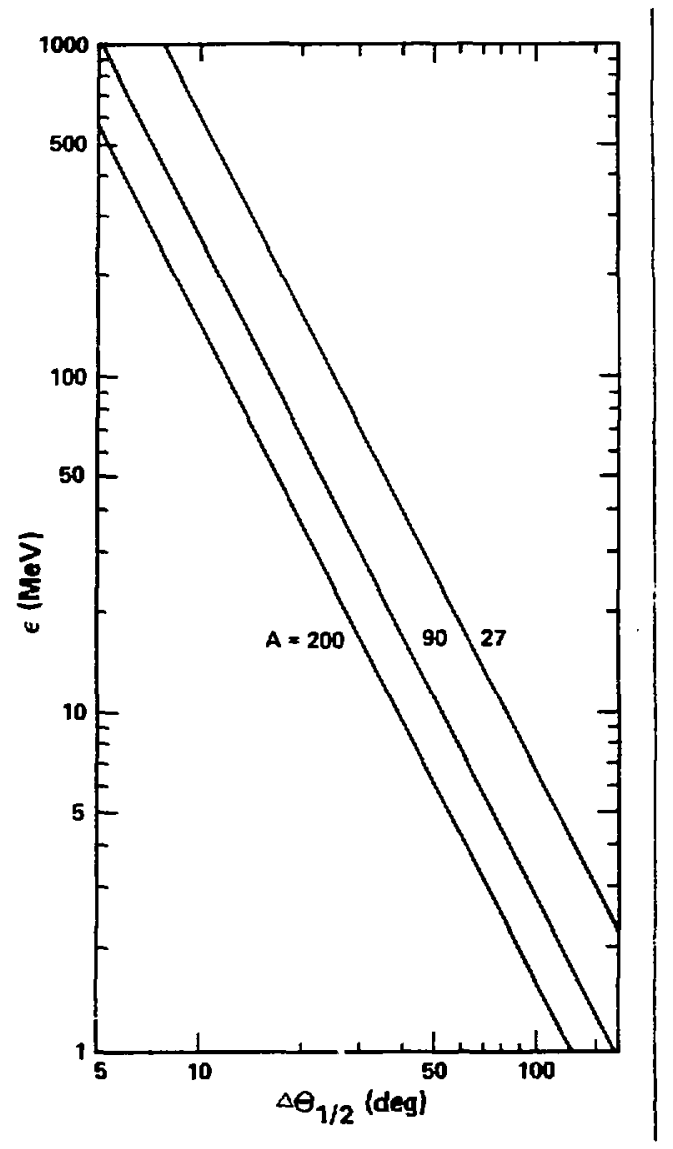

FIG. 12. Half angle versus nucleon energy due to the relationship of Eq. (6) for targets of $A=27,90$ and 200 . The radius used is the half density radius of the Myers' droplet model. Half angle refers to one half the angular opening symmetric about $0^{\circ}$.

models (including the GDH model) do not follow nucleon geometries. Therefore treatment of refraction in these models will require arbitrary procedures. It will be shown that diffraction limits will be comparable to or greater than expected refractive effects for some cases of interest and less in others. 


\section{PRECOMPOUND NUCLEON ANGULAR DISTRIBUTIONS}

We therefore approach these modifications to the $\mathrm{N}-\mathrm{N}$ scattering result with pessimism as to their physical content. Nonetheless, if it is possible to obtain an overall improvement in calculated angular distributions as reported in Ref. 12, the result is valuable for technology.

\subsection{Localization Considerations}

An important point which was emphasized by Mantzouranis et al. " is that the localization of the angular distribution resulting from the $\mathrm{N}-\mathrm{N}$ scattering kinematics is comparable to (sometimes less than, and sometimes greater than) the minimum value required by limits due to wave mechanical considerations. In Fig. 12 we show the minimum half angle for localization due to the relation given by Mantzouranis et al,

$$
R \Delta \theta>2 \pi / k \text {, }
$$

where $k$ is the nucleon wave number and where the sharp radius from the droplet model of Myers ${ }^{2}$ was used,

$$
R=1.18 A^{1 / 3}\left[1-1 /\left(1.18 A^{1 / 3}\right)^{2}\right]
$$

From this reslilt it may be seen, in particular for mucleons of the lower kinetic energies of interest (e.g. $\leq 20 \mathrm{MeV}$ ), that a semiclassical treatment of angular distributions as due to $\mathrm{N}-\mathrm{N}$ scattering kinematics is questionable. From this aiscussion and Fig. 12, we may anticipate a failure of the $\mathrm{N}-\mathrm{N}$ kernel approach due to localization constraints.

One semiclassical approach which has been investigated has been the addition of the semiclassical concept of refraction, as defined by Snell's law. If the failure is due to localization constraints, (diffraction) it is doubtrul that such a treatment (refraction) will provide a remedy whicil will scale over a wide 


\section{BLANN}

range of target mass and projectile/ejectile energies (see Fig. 12).

In the context of the above statement it is worthwhile to compare the single scattering result of Tamura et al. with the semiclassical single scattering kernel calculated with Eq. (4). This is done in Fig. 10 for the reaction ${ }^{200} \mathrm{~Pb}(p, n)$ for $45 \mathrm{MeV}$ incident proton energy and for exit channel energies of 26 and $36 \mathrm{MeV}$. The experimental results of Galonsky et al. are also shown. $^{22}$ The semiclassical result is for a Fermi gas with $E_{f}=40 \mathrm{MeV}$. The point of Fig. 10 is that the semiclassical result is definitely unable to reproduce the back angle yields for a single scattering event. From this comparison we may understand the deficiency as due to coherent processes not treated in the semiclassical approach. We may therefore immediately anticipate the inability of the $\mathrm{N}-\mathrm{N}$ scattering approach to reproduce data much beyond $90^{\circ}$ at those particle emission energies which result primarily from single scattering processes (the higher energies), as observed in the comparisons in the preceding section, and by all the work with this approach preceding this paper. We may see that quantum mechanical calculations which properly include refraction and diffraction are able to reproduce the experimental result, and we therefore understand the deficiencies and reasons for failure of the semiclassical result.

We next explore semiclassical modifications, purely ad-hoc in nature, to see if quantal effects may be incorporated in a simple manner into the $\mathrm{N}-\mathrm{N}$ scattering approach, as adjudged solely by success in reproducing experimental results. As will be discussed further on, quantial theories on this subject are not yet at as useful a stage of development as the simpler semiclassical models.

\subsection{Entrance channel refraction}

Here we discuss the possibility of increasing the intensity of mucleons scattered at large angies by considering refraction of the 


\section{PRECOMPOUND NUCLEON ANGULAR DISTRIBUTIONS}

incident and emitted particles. This was done by Hayakawa et al. for $(p, n)$ and $\left(p, p^{\prime}\right)$ reactions induced by $18 \mathrm{MeV}$ protons. ${ }^{14}$ Refraction and reflection were included in some intranuciear cascade codes, 5 and refraction has been treated in exciton model $\mathrm{N}-\mathrm{N}$ folding calculations by several authors (e.g., Ref. 12). We will first consider refraction in the entrance channel; this has some minimal justification since in the GDH model we can at least localize each entrance channel partial wave to a disk of cross sectional area $\pi X^{2} \mathrm{~T}_{\ell}(2 \ell+1)$ with impact parameter between $\ell$ and $(\ell+1)$. We will apply Snell's law as was done by Hayakawa et al. ${ }^{14}$ and by Costa et al. ${ }^{2}$ However, the justification for using this approach is not obvious when the incident particle wavelength is comparable to the nuclear skin thickness, sounding yet another word of caution.

A refractive index ' $n$ ' of the nucleus may be defined as

$$
\mathrm{n}=\mathrm{k}_{\text {in }} / \mathrm{k}_{\text {out }} \text {. }
$$

where $k_{\text {in }}\left(k_{\text {out }}\right)$ is the wave number of the projectile inside (outside) the mucleus and the refraction of a nucleon entering nuclear matter is given by Snell's law as

$$
k_{\text {out }} \sin \alpha=k_{\text {in }} \sin \beta \text {. }
$$

where Fig. 13 shows the angular relationships between incident and refracted waves.

In using Snell's law with the GDH model and N-N scattering we proceed in an iterative manner as follows: The average density along the particle (projectile) trajectory prior to iny refraction is calculated for impact parameter $\ell$. The corresponding Fermi energy $E_{F}^{\ell}$ for this density is calculated; the projectile binding energy $B$ is reduced by the ratio $E_{F}^{l} / E_{F}$, to an effective value $B_{\ell}$ where $E_{F}$ is the average Fermi 
M. BLANN

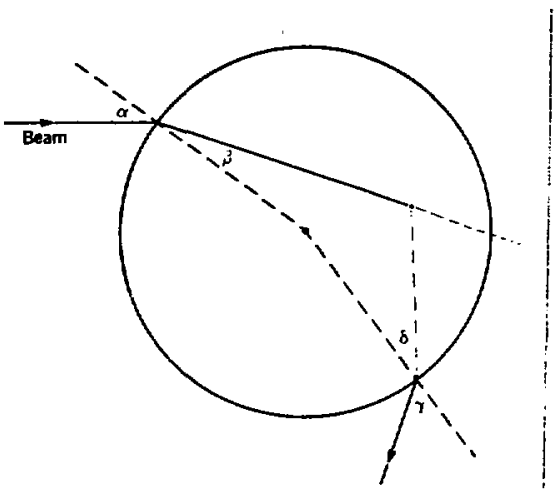

FIG. 13. Relationship of angle of incidence $(\alpha)$ and angle of refraction ( $\beta$ ) used in Snell's law as discussed in the text. The circle indicates one of a continuous number of possible collision sites along a nucleon trajectory. The exit incidence angle $\delta$ and angle of refraction $\gamma$ will depend upon the position of the collision, and on the scattering angle of the collision. Neither of these quantities is followed in exciton models of the type considered in this work.

energy of the nucleus, which is $30 \pm 2 \mathrm{MeV}$ for the parameters used in this work. An angle of refraction is computed for $K_{\text {in }}$ corresponding to an interior nucleon energy of $\varepsilon_{\text {out }}+B_{\ell}+E_{F}$ using Snell's law. A single iteration is then performed by calculating the average density of nuclear matter traversed by this refracted trajectory (in planar geometry with the original trajectory and nuclear center). Values of $E_{F}^{\ell}$ and $B_{\ell}$ are recalculated based on this result and the angle of refraction is recalculated. The same procedure is followed for an impact parameter of $(l+1)$, since the partial wave cannot be localized more closely. In Fig. 14 we show examples of incident proton refraction for $45 \mathrm{MeV}$ protons incident on a ${ }^{\circ} \mathrm{Zr}$ target. The partial cross sections of the various partial waves are shown as are the maximum kinematic single scattering angles for the arbitrary case of $35 \mathrm{MeV}$ emitted neutrons. 


\section{PRECOMPOUND NUCLEON ANGULAR DISTRIBUTIONS}

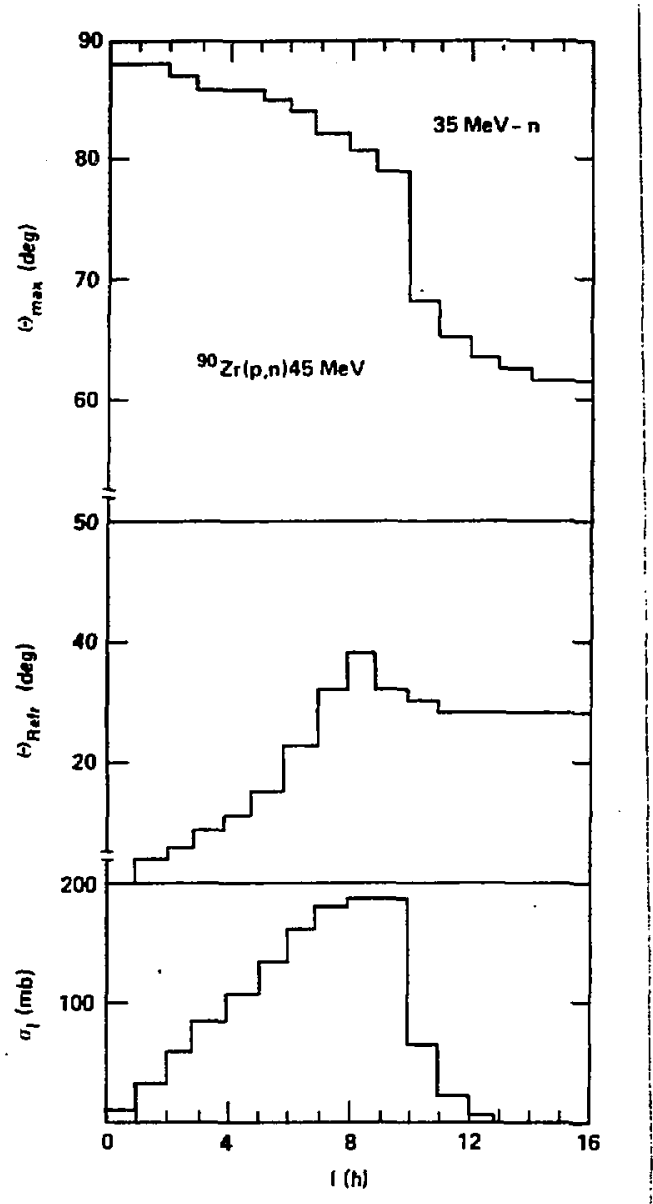

FIG. 14. Relationship between partial reaction cross sections (bottom), angle of refraction (center) and maximum kinematic scattering angle (top) for $45 \mathrm{MeV}$ mucleons incident on ${ }^{\circ} \mathrm{Zr}$. The bottom histogram gives partial reaction cross section (ordinate) versus incident proton angular momentum (I, abscissa). The angle of refraction for the entrance channel is shown versus $I$ in the center, calculated by the iterative process described in the text. The upper histogram shows the maximum kinematic single-scattering angle for $35 \mathrm{MeV}$ (laboratory frame) neutrons. The change in kinematic angles results from different Fermi energies resulting from the local density approximation of the geometry dependent hybrid model. 


\section{BLANN}

The kernel for N-N scattering is calculated for each impact parameter based on the value of $E_{F}^{2}$ calculated as described above. This 'bare' kernel is folded between the refractive angles described above for impact parameters $\ell$ and $(\ell+1)$. For additional intranuclear scattering events, a bare $\mathrm{N}-\mathrm{N}$ kernel based on a nuclear potential averaged over the entire nuclear volume is folded with the distribution for the refraction folded GDH result described above.

Results of including incident wave refraction are shown in Figs. 3 to $6,8,9$ and 25 . This procedure causes the calculated result to go to somewhat larger back angles before the onset of the rapid decrease with increasing angle, beyond which the calculation fails to reproduce experimental results. The change in agreement at forward angles is inconclusive; some results seem in better agreement with data at forward angles, whereas other results seem to predict too flat a distribution at forward angles, giving a result poorer than that from the pure N-N scattering result without the addition of spreading according to Eq. (8). Observing that there is still a major disagreement at very large back angles, we look for possible reasons to permit additional folding of the N-N scattering results. Again we must recognize the very ad-hoc, empirical nature of these procedures. We therefore next discuss refraction in the exit channel as a possible additional candidate for forcing semiclassically calculated results to further back angles, in a search for an empirical recipe to improve the overall success of the 'fit'.

\subsection{Entrance and exit channel refraction}

As discussed earlier, and as may be seen from Fig. 13, refraction in the exit channel is dependent not only on the entrance channel trajectory, but also on the position of the $\mathrm{N}-\mathrm{N}$ collision preceding particle emission and on the angle of 


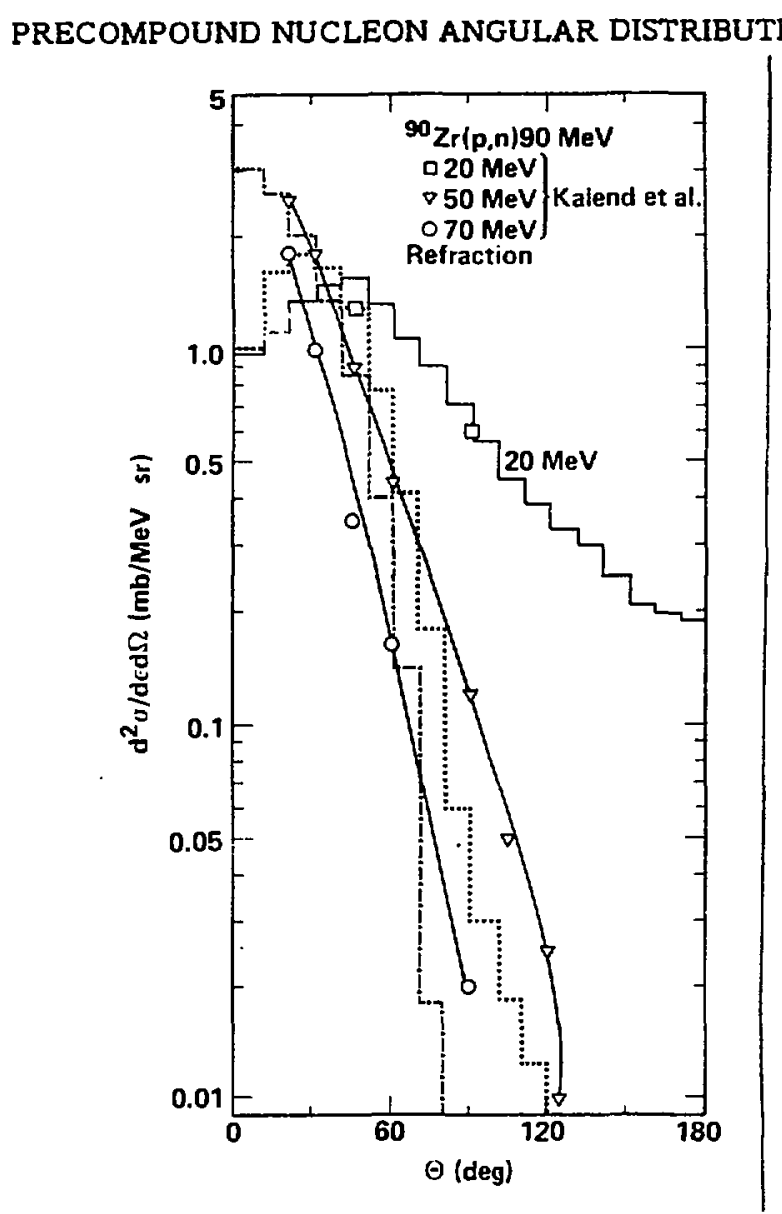

FIG. 15. As in Fig. 7, but calculated histograms include refraction in the entrance channel as described in the text.

scattering (i.e. the trajectory of the scattered particle). Most assuredly this is the information which is sacrificed in going from an intranuclear cascade representation of the reaction, (which is geometric in nature) to an exciton model representation (which is formulated in an energy space) where we have no geometric information of the type required in application of Snell's law. 


\section{BLANN}

The classical treatment of refraction in the entrance channel is modified by the Coulomb deflection a projectile proton experiences. It has been shown ${ }^{14}$ that in first order approximation the deflection is taken into account by replacirg Snell's law [Eq. (8b)] by

$$
\mathrm{K}_{\text {out }}^{\infty} \sin \alpha_{\infty}=\mathrm{K}_{\text {in }} \sin \boldsymbol{s}^{\infty}
$$

Here, $K_{\text {out }}^{\infty}$ denotes the wave number of the projectile at an infinite distance and $\alpha_{\infty}$ the angle between $\kappa_{\text {out }}^{\infty}$ and the normal of the nuclear surface at the point of incidence.

Coulomb repulsion leads to $\alpha_{\infty}<\alpha$; the refractive index [Eq. (8a)] is reduced by the factor $K_{\text {out }} / K_{\text {out }}^{\infty}<1$. These opposite effects in part cancel. At some tens of $\mathrm{MeV}$ projectile energy the reduction in angle $\alpha_{\infty}<\alpha$, although small, ${ }^{5}$ dominates. In this sense entrance channel refraction without deflection gives an upper limit, and this is why we do not consider the effect of Coulomb deflection in the remainder of this paper.

We note in Fig. 12 that the minimum localization angles for particles in the entrance channel are roughiy comparable to the angles of refraction as calculated by Snell's law [compare Fig. (14)]. We therefore recomputed the angular distributions calculated with Snell's law for the entrance channel only, using the $\Delta \theta$ of Fig. 12 in place of the result of Snell's law. The two results were very similar for all systems compared. We therefore used Fig. 12 results [i.e., the relationship of Eq. (6)]) to fold once more the angular distribution of particles in the exit channel. Some improvement resulted over the 'entrance channel only' results, but some back angle discrepancy remained. We therefore arbitrarily reduced the radius of $\mathrm{Eq}$. (7) from the $50 \%$ density value to the $90 \%$ density value (by reducing the $R$ of Eq. (5) by $1.1 \mathrm{fm}$ ) to give some additional improvement at back angles. 


\section{PRECOMPOUND NUCLEON ANGULAR DISTRIBUTIONS}

Results of calculations treating 'refraction' in the entrance and exit channels (via the relationship of Eq. 6 which might better be called a diffraction result) may be seen in Figs. 16-18. In all cases we observe that this results in an over-correction at forward angles with too flat a shape and too low a cross section. At the lower incident and exit channel energies there is some improvement in fitting back angles, but for the case of ${ }^{\circ} \mathrm{Zr}(\mathrm{p}, \mathrm{n})$ with $70 \mathrm{MeV}$ exit channel energy, for which semiclassical approaches should be most valid, we see that an over correction resuits at the back angles as well. It is our opinion, based on the results presented herein, that our attempts to add semiclassical approximations to account for quantal phenomena fails to give a uniform improvement in the angular distribution calculation based on the nucleon-nucleon (N-N) scattering approach.

Having made perturbations to the results of the $\mathrm{N}-\mathrm{N}$ scattering angular distribution by our semiclassically added estimates of quantal effects, it is valuable to see if the modified angular distributions result mainly from the $\mathrm{N}-\mathrm{N}$ scattering kernel, mainly from the additions to approximate quantal phenomena, or if they are somewhat sensitive to both.

In order to get a feeling for the answer to this question we re-computed several angular distributions with 'refraction' in the entrance and exit channels as before, but we set the $\mathrm{N}-\mathrm{N}$ scattering kernel to zero at all angles but $45^{\circ}$ in one set of calculations (Figs. 16, 17), and in yet another set of calculations we set the $\mathrm{N}-\mathrm{N}$ scattering kernel to zero at all angles except $0^{\circ}$. i.e., the assumption that there is no N-N scattering (Figs. 16-18). From this exercise we can make several observations. First, there is little Alfference between the refraction folded result using the full $\mathrm{K}-\mathrm{K}$ scattering kernel of Eqs. 4-5, and the result assuming that all $\mathrm{N}-\mathrm{N}$ scattering is at $45^{\circ}$. Sensitivity to the 


\section{BLANN}

details of the $\mathrm{N}-\mathrm{N}$ scattering kernel may be seen to be mostly lost when the 'refraction' is added to the calculation.

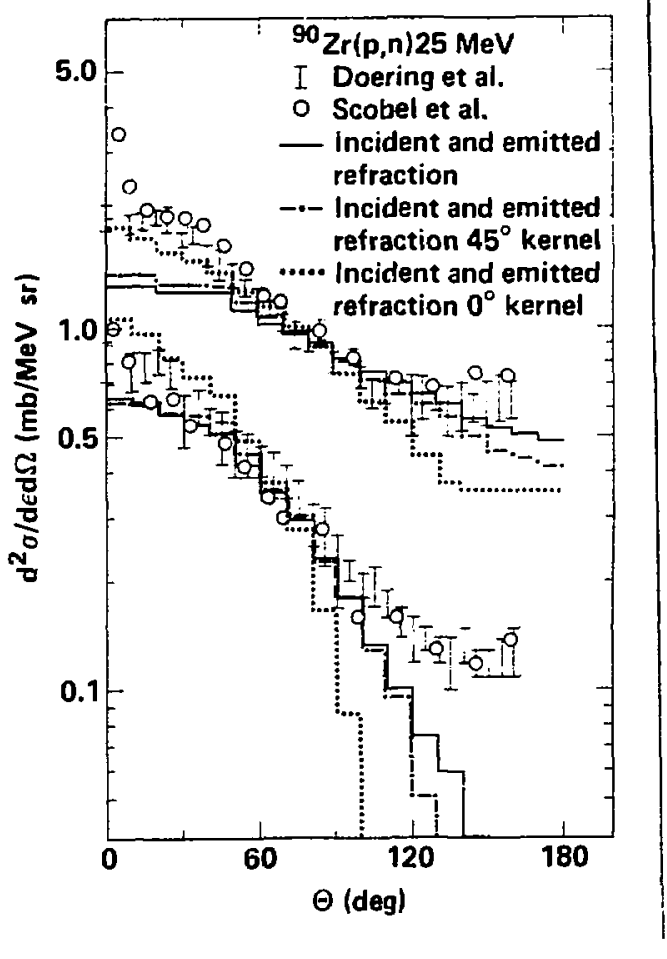

FIG. 16. Angular distributions for $10 \mathrm{Zr}(\mathrm{p}, \mathrm{n})$ for $25 \mathrm{MeV}$ incident protons, 9 and $14 \mathrm{MeV}$ exit channel neutrons. Data are as in Fig. 5. The solid histogram is the folded $N-N$ scattering calculation which includes folding for refraction in both entrance and exit channels as described in the text. The dot-dash histogram is as the solid histogram, except that the $\mathrm{N}-\mathrm{N}$ scattering kernel is replaced by one which is zero at all angles except $45^{\circ}$. The dotted histogram is as for the solid histogram with the N-N kernel zero at all angles except $0^{\circ}$, 1.e., only the ad-hoc 'refractive' effects are represented in the folded result. 
PRECOMPOUND NUCLEON ANGULAR DISTRIBUTIONS

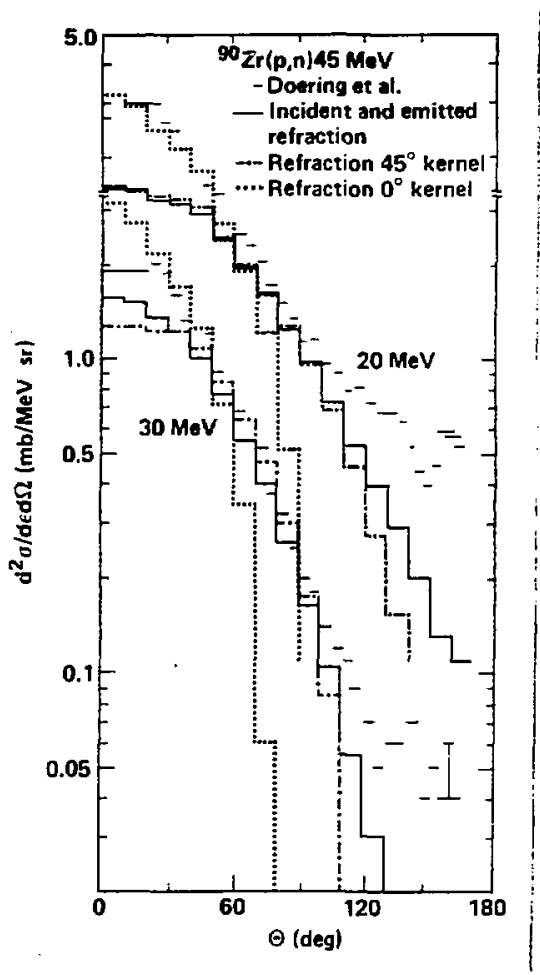

FIG. 17. As in Fig. 16 for $45 \mathrm{MeV}$ incident proton energy.

Considering the result where we assume no $\mathrm{N}-\mathrm{N}$ scattering, we get a better overall fit to data for ${ }^{\circ} \mathrm{Zr}(\mathrm{p}, \mathrm{n})$ at $25 \mathrm{MeV}$ incident energy (Fig. 16) and $9 \mathrm{MeV}$ exit energy over the result using the full $\mathrm{K}-\mathrm{K}$ kernel. At 45 and $90 \mathrm{MeV}$ incident energy a stronger case may be made for the necessity of some additional mechanism to broaden the angular distribution - which we of course believe is the $\mathrm{N}-\mathrm{N}$ scattering. There are different interpretations which may be placed on these observations. One we would keep in mind is that the main message from these and earlier observations in this work (see Figs. 10 and 12 


\section{BLANN}

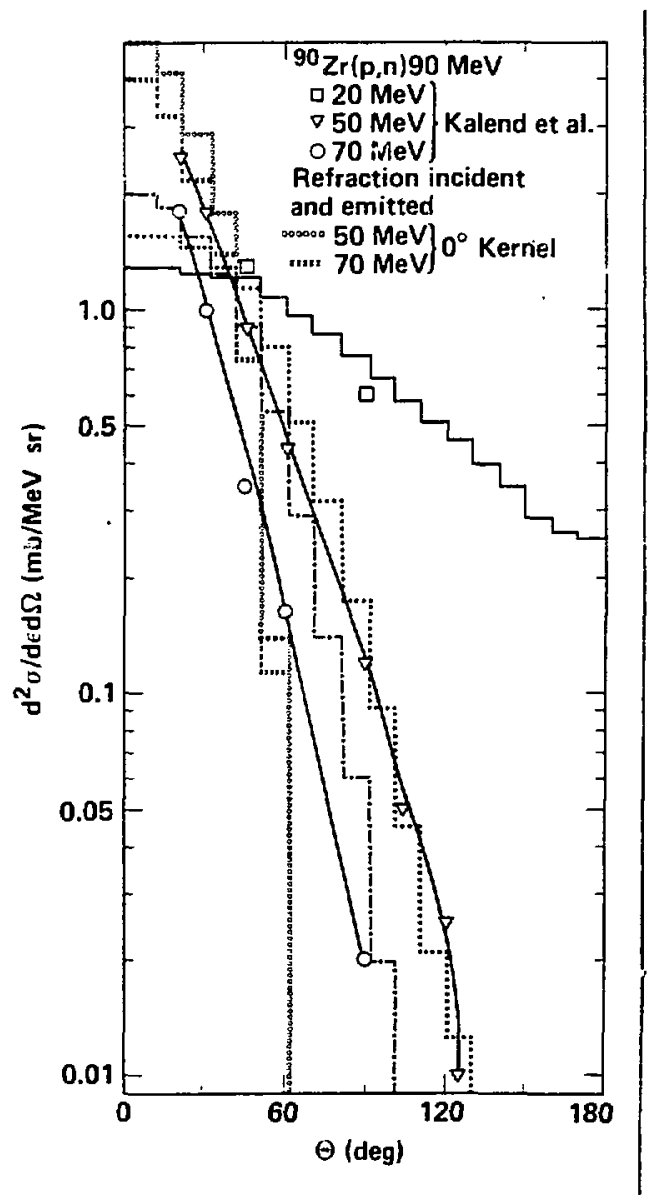

FIG. 18. As in Fig. 15, but an additional folding is performed for refraction in the exit channel. Results are shown at 50 and 70 MeV exit chamnel energy using a $N-N$ kernel which is zero at all angles except $0^{\circ}$. 


\section{PRECOMPOUND NUCLEON ANGULAR DISTRIBUTIONS}

and discussions) is that at the lower bombarding energies the angular distributions owe their form more to quantal considerations than to the results of the N-N scattering process. If this is so it may be that semiclassical modifications are unable to approximate these wave-mechanical results, or alternatively that an adequate semiclassical approximation has not as yet emerged. We stress that these are our conclusions based on the analyses of this work. Broader analyses of better data sets could cause a re-evaiuation of these conclusions, and other authors doubtless do not share these viewpoints.

Having emphasized a very cautious approach in concluding what we have learned about the physics involved in the continulis n angular distributions under discussion, we should also re-emphasize the positive side: the semiclassical approach reproduces the forward part of the angular distribution (and therefore the major part of the cross section) quite well, using only a folded $\mathrm{N}-\mathrm{N}$ scattering kernel. This success is achieved by combining the work of Goldberger ${ }^{-12}$ and of Hayakawa et al., ${ }^{2,1 \text { * }}$ with the concept due to Mantzouranis et al." of using an exciton model approach to fold successive kernels. This result is easily calculable and useful for nuclear technology.

While we feel that the answer to the back angle yields requires quantum mechanical treatments, we have seen no convincing evidence that such approaches are as yet at a stage that accurate a-priori predictions of angular distributions can be made. For example, the approacis of Tamura et ai. has been shown to work well for incident proton energies up to $62 \mathrm{MeV}$. However in Fig. 19 we show results of the first step only calculation for the anglalar distribution of neutrons following $90 \mathrm{MeV}$ proton bombardment of ${ }^{10} \mathrm{Zr}^{2 *}$ The $20 \mathrm{MeV}$ result required significantly different parameters than the 50 and 70 MeV results. The $20 \mathrm{MeV}$ calculated result of Tarnura is 
M. BLANN

plotted multiplied by 165 , whereas the 50 and $70 \mathrm{MeV}$ results are plotted multiplied by five. The results of the semiclassical calculations presented in this work are also repeated in Fig. 19. all values plotted without renormalization. These results are at least as good as the quantum mechanical results of Tamura with respect to shape of the angular distributions, and are better in predicting the absolute magnitude of the experimental results. The theory of Tamura requires, at the least, fitting to experimentally measured discrete transitions sefore magnitudes of continuur. angular distributions may be calculated.

In Fig. 20 we show results of INC calculations which include reflection and refraction in the exit channel. ${ }^{20}$ Here the back angle agreement is improved over results without reflection/refraction, but back angle yields are still inadequate. This figure serves to lllustrate that the exciton model with ad-hoc addition of refraction gives results qualitatively consistant with the INC model.

\subsection{Additional possible causes of back angle scattering in the} semiclassical framework.

A model for precompound angular distributions due to Feshbach et al.' (FKK) suggests that two classes of exciton configurations should be considered during the equilibration process, multistep direct (MSD) and multistep compound (MSC). In the former, one (or more) scattered particles are always in the unbound continume. In the latter, all particles are bound for at least one exciton configuration preceding emission. For the MSD componert a quantum mechanical calculation is performed predicting a forward peaked angular distribution. For the MSC component it is assumed that the angular distribution will be 


\section{PRECOMPOUND NUCLEON ANGULAR DISTRIBUTIONS}

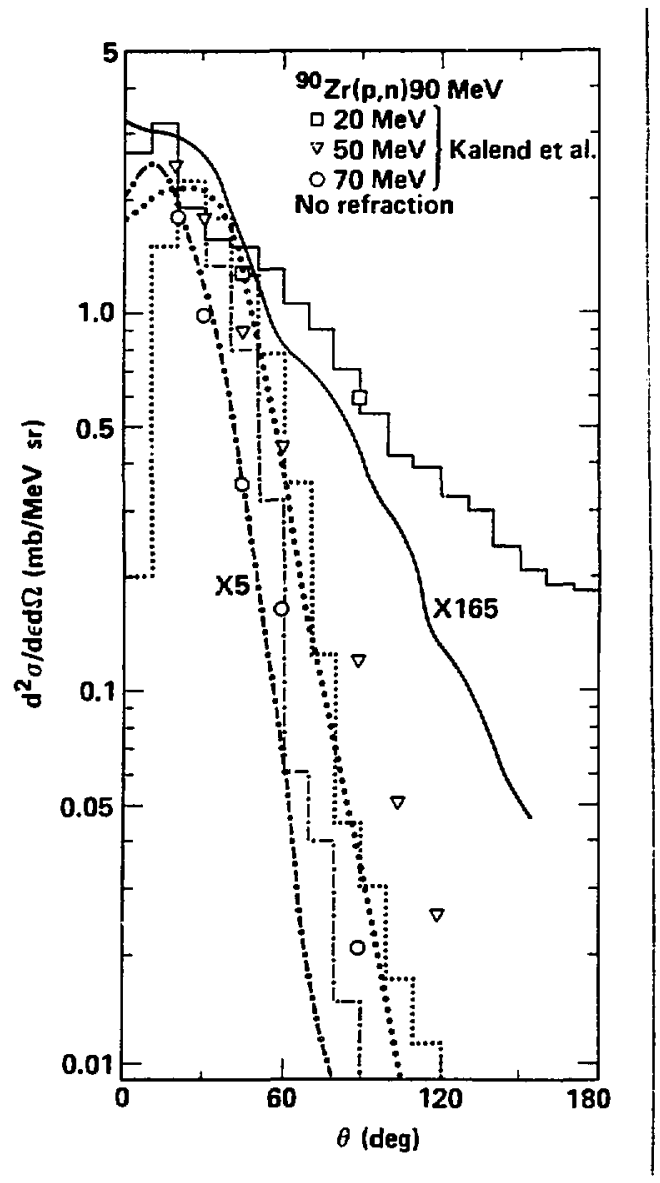

FIG. 19. Angular distributions for the $10 \mathrm{Zr}(\mathrm{p}, \mathrm{n})$ at $90 \mathrm{MeV}$ incident proton energy. The histograms are results of the folded N-N scattering calculation as in Fig. 7. The heavy solid, heavy dotted, and heavy dot-dash curves are results of the one step scattering calculation using the theory of Tamura et al." The $50 \mathrm{MeV}$ and $70 \mathrm{MeV}$ results are shown increased fivefold over the calculated result; the $20 \mathrm{MeV}$ result has been multiplied by 165 . These adjustments were made to facilitate comparisons between calculated and experimental shapes. All semiclassical results presented in this work were plotted without renormalization. 
M. BLANN

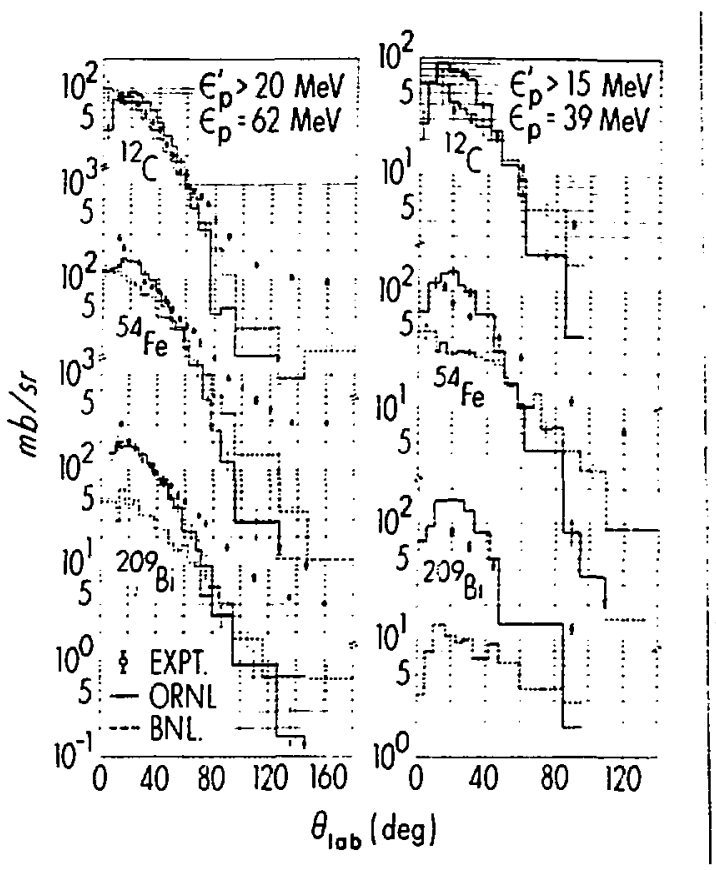

FIG. 20. Calculated (INC) and experimental angular distributions for (p,p') reactions on $12 \mathrm{C}, 14 \mathrm{Fe}$, and $209 \mathrm{Bi}$ targets. These results are from Ref. 29. The solid histogram (ORNL) is from an INC code without refraction; the dashed histogram (BNL) is from a code which includes reflection and refraction.

symmetric about $90^{\circ}$ in the center of mass due to random relative phases of matrix lements involving different total angular momenta.

To the extent that the MSC contribution is treated as a free parameter in calculations with the FKK model, a fit to the back angle data is guaranteed. Indeed if such a component were invoked in the semiclassical $N-N$ scattering results (without refraction) a reasonable fit could be obtained for all spectra shown in this work. 


\section{PRECOMPOUND NUCLEON ANGULAR DISTRIBUTIONS}

Fortunately or unfortumately we may use the exciton model enerbl partition functions to estimate the fraction of configurations for which all particles are bound. If the least tightly bound particle has binding energy $B$, and the excitation energy is $E$, then the result for the 3 exciton configuration is

$$
F_{M S C}=(B / E)^{2} .
$$

This expression could easily be modified for finite well depth, however the limit given in Eq. (9) is sufficient for our purposes. Particle emission for subsequent configurations clearly cannot exceed an energy of $2 \mathrm{~B}+\mathrm{E}_{\mathrm{f}}$ for MSC components following the 3 exciton configuration. A higher limit applies to higher exciton numbers, but their contribution decreases so rapidly that they may safely be ignored.

We have used Eq. (9) to estimate a MSC component under the assumption that Eq. (9) gives the fraction of the precompound cross section at each channel energy which is emitted isotropically. The addition of this component greatly improves the agreement of calculated and experimental spectra in Figs. 6-12; however the MSC component is still too small to fully reproduce the back angle data. More importantly though, an additional two body interaction is required to direct sufficient energy on a particle exciton for emission, so that only a very small fraction of our estimated upper limit should actually be realized in the exit channel. Given the cross section as a free parameter, as in some applications of the model of Ref. 7, we can reproduce the data very well. Making more realistic estimates we feel that this component cannot reasonably explain the general back angle deficiency.

Recent work by Bonetti and Colli-Milazzo, 30 using the tileory of Ref. 7 has however made a good case for the 


\section{BLANN}

importance of the multi-step compound contribution in some regions of the spectra. These calculations use a two body force which is consistenc with that used for the multi-step direct component. This result seems to be an encouraging. large step forward in removing some of the objections stated in this subsection. However the point that the semi-classically calculated back angle deficiency at higher energies cannot be reproduced via the MSC component is reinforced by the work of Bonetti and Colli-Milazzo.

\subsection{Possible improvements of semiclassical model for fitting back angle daca.}

There are several factors not included in the calculations of this work which might modify the back angle part of the semiclassical N-N scattering calculation which should be stated, some of which warrant further investigation:

(1) Isotropic $\mathrm{N}-\mathrm{N}$ scattering angular distributions were assumed in the work of Hayakawa et al. ${ }^{24}$ as used in this work: Experimentally there is considerable forward-backward peaking over much of the energy range we have considered. ${ }^{31}$

(2) A maximum value of the Fermi energy of $40 \mathrm{MeV}$ was used in the $\mathrm{N}-\mathrm{N}$ scattering calculations in this work. A higher momentum component would increase back angle scattering (this effect may be seen in Fig. 14, where (going in the opposite direction) the maximum scattering angle decreases as the average $E_{f}$ decreases with increasing impact parameter).

(3) The kinematics assumed in the $\mathrm{N}-\mathrm{N}$ scattering calculation are two-body. A small admixture of the projectile nucleon interacting with e.g., a nucleon pair would grossly alter the back-angle distribution of the kernel we used which includes only two body scattering processes. Nucleon interactions with 


\section{PRECOMPOUND NUCLEON ANGULAR DISTRIBUTIONS}

larger clusters would have a similar result; the limit of this effect is nuclear scattering with the entire target nucleus, which was discussed in previous subsections.

Let us consider these items in order. The first could result in up to factors of three in intensity at extreme forward and backward angles, (before Pauli principle reduction) but would not modify the kinematic back angle limit for a single $\mathrm{N}-\mathrm{N}$ scattering process. Much of the failure to reproduce back angle results comes from this kinematic limit. A more realistic $\mathrm{N}-\mathrm{N}$ angular distribution might therefore give better agreement to slightly larger angles, but the main precipitate decrease in calculated cross sections at large angles would not change in angle.

Item (2) should próbably be classificd as extremely unlikely to be relevant. Rough estimates of high nucleon momentum components suggest that significant contributions to the $N-N$ scattering result due to this cause would be unrealistic.

Item (3) is well worth consideration. The assumption of two body scattering is dependent upon the $\mathrm{N}-\mathrm{N}$ collision period being short with respect to the period between collisions. This problem was recently considered by Kawai and Weidenmiller. ${ }^{32}$ Their estimates showed that while the $\mathrm{N}-\mathrm{N}$ collision period was less than the inter-collision period for the energies considered in this work, it was not so much shorter as to render multiple body collision processes negligible. An admixture of $10-20 \%$ of such processes would not significantly alter the predicted angle integrated spectra, yet they could very considerably modify the assumed intranuclear scattering kernel with respect to kinematic back angle limits. This is probably an important class of processes to consider if one is to adequately explore the limitations of the semiclassical approach in reproducing back angle scattering results. ${ }^{1 *}$ 


\section{BLANN}

\section{CONCLUSIONS}

In this work we have applied the analytic solution of the angular distribution of nucleons following the scattering of a projectile nucleon with nucleons of a Fermi gas. An isotropic nucleon-nucleon scattering angular distribution was assumed in deriving the analytic expression, and the Pauli exclusion principle was included. While the equations used were taken from the work of Kikuchi and $\mathrm{Kawai}^{2}$, earlier expositions are to be found in the work of Goldberger $^{13}$ and of Hayakawa, Kikuchi and Kawai ${ }^{14}$. One important observation is that the shape of the single scattering kernel resulting from the approach is in much better agreement with experimental precompound angular distributions than results using the stationary target nucleon approximation, as shown by Costa et al. ${ }^{12}$

Following the example of Mantzouranis et a1.," we have used an exciton model formulation to give the spectral contributions as a function of the number of intranuclear scattering events (the so-called exciton hierarchy). We have however used the geometry dependent hybrid model ${ }^{1}$ which retains somewhat more of the reaction information which may be relevant to angular distributions than pure exciton formulations (such as the hybrid model) which are averaged over the additional geometric details, making local density dependent treatments more tenuous.

We have used this approach to compare with data spanning a fairly broad range of incident nucleon energies (14.7 to $90 \mathrm{MeV}$ ) and exit channel energies ( 9 to $70 \mathrm{MeV}$ ). The folded $\mathrm{N}-\mathrm{N}$ scattering kernel (which is mostly the single scattering result when exit channel energy is more than half the entrance channel energy) reproduces this broad range of results very well at angles less than $\sim 90^{\circ}$ and greater than $20^{\circ}$; this region includes the major part of the cross section. As with earlier related 


\section{PRECOMPOUND NUCLEON ANGULAR DISTRIBUTIONS}

treatments such as the INC, angles in excess of $90^{\circ}$ are, for many of our examples, underestimated. The GDH model results are in better agreement with data than results of the hybrid model.

We have compared the single scattering result of Tamura et al.' with our semiclassical result and see that quantum mechanical treatments provide back angle cross sections which are kinematically forbidden in the semiclassical $\mathrm{N}-\mathrm{N}$ scattering kernel. This result and a simple comparison with angular localization constraints due to Eq. (6) lead us to the conclusion that understanding of the back angle yields requires quantum mechanical formulations, a conclusion in agreement with Tamura et al. ' and with Feshbach et al. "However the comparisons of Fig. 19 indicate that these approaches may still be poorer than the much simpler semiclassical treatments when a-priori predictions are required, and for applications in nuclear technology the semiclassical approach is surely vastly easier to perform. We should, however, emphasize the excellent results being obtained by Bonetti using the formalism of Feshbach et al.; note for example Fig. 21 from a recent work by Scobel et al. ${ }^{33}$

We have investigated ad-hoc methods of trying to join quantum mechanics with classical mechanics via 'refraction' and/or folding due to Heisenberg constraints. While we can force a fit to back angle yields at lower projectile energies by this method, we find that at the higher bombarding energies where the assumptions of refraction should be best, the results show an over correction at forward angles, and poorer shapes overall. We therefore are sikeptical of even the empirical efficacy of such approaches, while agreeing that quantal effects are the major missing physics in using the semiclassical $\mathrm{N}-\mathrm{N}$ scattering kernel. If there is to be a legitimate method of increasing calculated back angle yields in the semiclassical approach it is likely that some 


\section{BLANN}

contribution from multiple excitation modes (other than pure nucleon-nucleon interactions) should be considered together with nucleon-nucleon interactions.

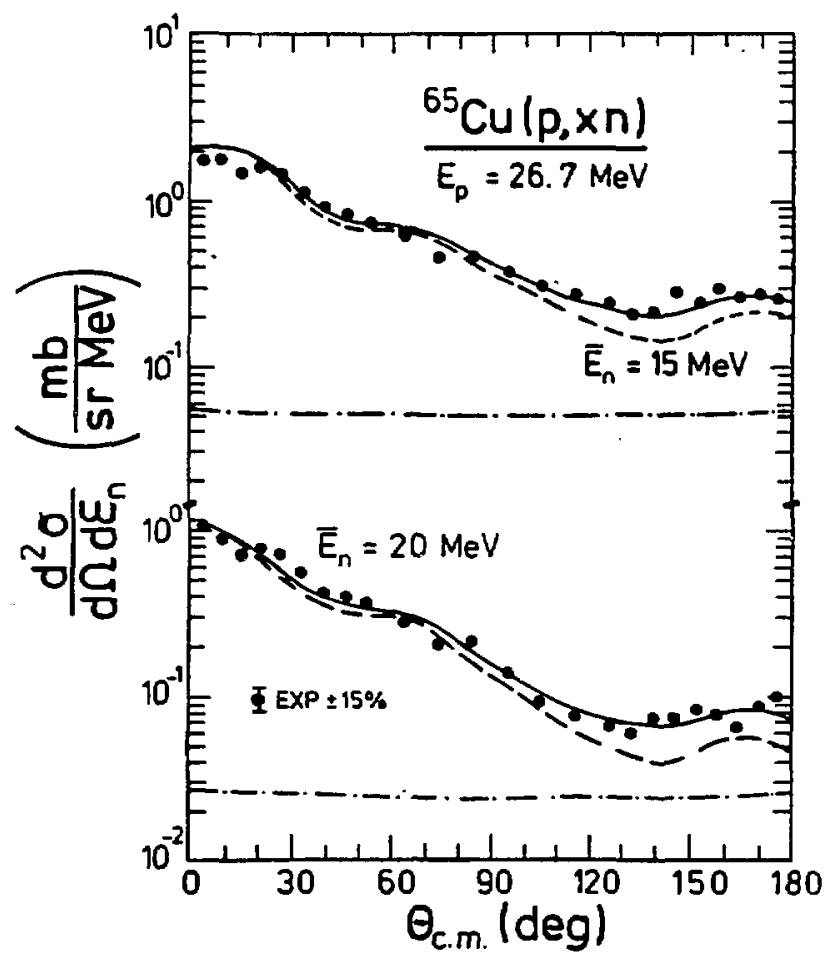

FIG. 21. Experimental (solid points) and calculated angular distributions for proton (26.7 MeV) induced reactions on ${ }^{\prime s} \mathrm{Cu}$. The dashed curve is the prediction by Bonetti of the multi-step direct component of the theory of Ref. 7; the solid line is the sum of calculated multi-step direct plus multi-step compound reactions. 


\section{BLANN}

Finaliy the experimental results should not escape scrutiny. Many of the data cited in this work did not include adequate background measurements at back angles, since the purpose of the experiments did not include special focus on back angle yields. If we are to focus great attention on fitting the very small portion of the cross sections at far back angles, some data sets extending as close to $180^{\circ}$ as possible, with careful attention to minimizing and correcting for background at these back angles, would be valuable and indeed indispensible to final evaluation of possible models. Additional measurements extending toward $0^{\circ}$ would similarly be valuable, as the comparisons of this and other works show this to be another region where the pure N-N scattering kernel may be inadequate in reproducing details of the angular distributions.

\section{ACKNOWLEDGEMENTS}

One of the authors (M.B.) appreciates valuable discussions with Profs. A. Kerman, T. Tamura, and F. Petrovich. We appreciate also the calculations provided us for Fig. 19 by Prof. Tamura and by $\mathrm{Mr}$. M. Benhamow. We appreciate a critical reading of the manuscript and helpful suggestions from Drs. H. Gruppelaar and H. Akkermans. Work performed under the auspices of the U.S. Department of Energy by the Lawrence Livermore National Laboratory under contract number W-7405-ENG-48.

\section{REFERENCES}

1. M. BLANN, Phys. Rev. Lett 28, 757 (1972).

2. K. KIKUCHI and M. KAWAI, "Nuclear Matter and Nuclear Reactions," North Holland, Amsterdam (1968).

3. R. SERBER, Phys. Rev. 72, 1114 (1947); N. METROPOLIS et a1., Phys. Rev. 110, 185, 204 (1958).

4. H. W. BERTINI, Phys. Rev. 131, 1801 (1963). 
PRECOMPOUND NUCLEON ANGULAR DISTRIBUTIONS

5. K. CHEN et al., Phys. Rev. 166, 949 (1968); K. CHEN, G. FRIEDLANDER and J. M. MILLER, Phys. Rev. 176, 1208 (1968); K. CHEN, G. FRIEDLANDER, G. D. HARP and J. M. MILLER, Phys. Rev. C 4, 2234 (1971).

6. T. TAMURA, T. UDAGAWA and H. LENSKE, Phys. Rev. C 26, 379 (1982).

7. H. FESHBACH, A. KERMAN and S. E. KOONIN, Ann. Phys. (NY) 125, 429 (1980); for use of this theory see R. BONETTI, M. CAMNASIO, L. COLLI-MILAZZO and P. E. HODGSON, Phys. Rev. C24, 71 (1981).

8. C. MANTZOURANIS, H. A. WEIDENMULLER and D. AGASSI, Z. Phys. A276, 145 (1976).

9. Sun ZIYANG, Wang SHUNUAN, Zhang JINGSHANG and Zhuo YIZHONG, Z. Phys. A305, 61 (1982).

10. J. M. AKKERMANS, H. GRUPPELAAR and G. REFFO, Phys. Rev. C 22, 73 (1980).

11. J. M. AKKERMANS, Phys. Lett. 82B, 20 (1979).

12. C. COSTA, H. GRUPPELAAR, and J. M. AKKERMANS, Phys. Rev. C 28, 587 (1983).

13. M. L. GOLDBERGER, Phys. Rev. 74, 1269 (1948).

14. S. HAYAKAWA, M. KAWAI, and K. KIKUCHI, Prog. Theor. Phys. 13, 415 (1955).

15. 1. UDAGAWA, K. S. LOW, and T. TAMURA, Phys. Rev. C 28 . 1033 (1983); T. TAMURA, private communication (1983).

16. A. KERMAN, private communication (1983).

17. M. BLANN and H. K. VONACH, Phys. Rev. C 28, 1475 (1983).

18. W. D. MYERS, Droplet Model of Atomic Nuclei, (IFI Plenum, New York, 1977).

19. M. BLANN, Nucl. Phys. A213, 570 (1973).

20. G. MANTZOURANIS, Phys. Lett. 63B, 25 (1976).

21. 10c. cit. Ref. 10; in this work (n,n') spectra from $14.7 \mathrm{MeV} n$ induced reactions were summed for $a$ bin between 2 and 11 $\mathrm{MeV}$. The spectra below $7 \mathrm{MeV}$, which dominate the cross sections, correspond to 'slow' particles. Refs. 10 and 20 are only a few of many examples 'where the 'fast' particle requirement of Ref. 8 has been relaxed or ignored.

22. A. GALONSKY, R, R. DOERING, D. M. PATTERSON, and $H$. W. BERTINI, Phys. Rev. C 14, 748 (1976).

23. D. HERMSDORF et al., Report ZfK-277 (Rossendorf bei Dresden, 1974); A. TAKAHASHI et al., Osaka Univ. Oktavian Report A-83-01 (1983) unpublished; J. L. KAMMERDIENER, Lawrence Livermore National Laboratory, Livermore, CA, UCRL-51232 (1972) unpublished.

24. A. MARCINKOWSKI, et a1., Nucl. Sci. Eng. 83, 13 (1983); A. MARCINKOWSKI et a1., unpublished. 
M. BLANN

25. M. BLANN, R. R. DOERING, A. GALONSKY, D. M. PATTERSON, and F. E. SERR, Nucl. Phys. A257. 15 (1976).

26. W. SCOBEL et al., Phys. Rev. C 30, 1480 (1984).

27. A. M. KALEND et a1., Phys. Rev. C 28, 105 (1983).

28. T. TAMURA and $M$. BENHAMOW, private communication (1983).

29. H. W. BERTINI, G. D. HARP, and F. E. BERTRAND, Phys. Rev. C 10, 2472 (1974).

30. R. BONEITI, private communication (1984); R. BONETTI and L. COLLI-MILAZZO, reported at workshop on Coincident Particle Emission From Continuum States; Bad Honnef, F.R.G. (1984).

31. R. WILSON, "The Nucleon-Nucleon Interaction", Interscience Tracts on Physics and Astronomy no. 16, Ed. R. E. Marshals, Interscience (New York) 1963.

32. M. KAWAI and H. A. WEIDENMULLER, Z. flir Physik A 311 , 117 (1983). 